\title{
共轭烯酸与聚乙二醇缩合反应研究进展
}

\author{
陈锦芳许孝良*李小年*
}

(浙江工业大学 化学工程学院 工业催化研究所 杭州 310014)

\begin{abstract}
摘要 聚乙二醇不饱和酸酯是一类重要的高聚物单体，其与其他单体接枝共聚也可合成多功能化的高分子聚合物. 主 要综述了聚乙二醇与丙烯酸、甲基丙烯酸、马来酸等共轭烯酸缩合反应的研究进展, 并对其发展进行了展望.

关键词 共轭烯酸; 聚乙二醇; 缩合反应
\end{abstract}

\section{Research Development on the Condensation Reaction of Conjugated Olefine Acid and Polyethylene Glycol}

\author{
Chen, Jinfang $\quad \mathrm{Xu}$, Xiaoliang* $\quad \mathrm{Li}$, Xiaonian* \\ (Institute of Industrial Catalysis, College of Chemical Engineering, Zhejiang University of Technology, Hangzhou 310014)
}

\begin{abstract}
Unsaturated acid esters of polyethylene glycol (PEG) are important monomers for the preparation of polymers. In addition, multifunctional polymers can be synthesized by the graft copolymerization of unsaturated acid esters of PEG with other monomers. The research development of the condensation reaction of PEG with conjugated olefine acids, such as acrylic acid, methyl acrylic acid, maleic acid, etc. is reviewed, and its outlook in the future is given.
\end{abstract}

Keywords conjugated olefine acid; PEG; condensation reaction

近年来共轭不饱和酸与聚乙二醇(PEG)的缩合反应 广泛地引起了人们的关注. PEG 接枝不饱和酸所形成的 酯是一类重要的高分子单体, 由于分子中含有酯基、羧 基和不同长度的聚氧乙烯基等基团，使其具有许多优良 的性能, 在水凝胶、涂料、胶粘剂、表面活性剂和超塑 化剂等领域得到了广泛的应用 ${ }^{[1 \sim 3]}$.

PEG 末端含有两个活性羟基，在接枝不饱和酸的过 程中可以通过控制反应条件，合成同时含有不饱和键、 酯基和羟基的单酯化物，以及具有两个不饱和键和酯基 的双酯化物. PEG 的单酯化单体末端含有的羟基和双键, 使它具有亲水性强、可聚合性高以及聚合后呈梳状结构 等特点. 以它作为单体与(甲基)丙烯酸、马来酸䣶等不 饱和酸共聚生成的高聚物是高效第三代减水剂的重要 部分. 与传统的菜磺酸盐类、木钻类、密胺系减水剂相 比, 该类减水剂含有羟基、羧基、长的聚氧乙烯链以及 特殊的梳状结构, 在水泥混凝土中表现出较强的亲水性 和较大的空间位阻, 使得水泥混凝土的流动性更好、抗 压能力更强、坍落度更低, 因而引起越来越多的学者的
重视.

PEG 的双酯化单体末端含有两个不饱和基团，与不 同的活性不饱和单体共聚成交联网状结构的高分子聚 合物. 这种高聚物的特殊的支架结构，与聚氧乙烯基的 强吸附性一起, 决定了其作为生物水凝胶的重要作用. 天然衍生物凝胶如壳聚糖、纤维蛋白、胶原质、动物胶 等稳定性差、易降解，而该类合成凝胶具有可再生性、 吸附性强、生物相容性好、可控性高等特点，广泛应用 于药物、蛋白质、DNA 的传递系统. 作为药物载体, 这 类水凝胶不仅克服了前体药物生物药效低、降低人体免 疫力、循环周期长等缺点, 其特殊网状结构还表现出较 好的生物适应性，减少了炎症、血栓以及对组织的损坏.

本文对 PEG 与各种不同的不饱和羧酸的缩合反应 做出简单的归纳, 并对其发展进行了展望.

\section{1 丙烯酸}

由于聚乙二醇(PEG)支链具有较好的极性、水溶性 和柔性使得丙烯酸(AA)与 PEG 缩合所得的酯化产物广

*E-mail: xuxiaoliang@zjut.edu.cn; xnli@zjut.edu.cn

Received July 14, 2014; revised September 12, 2014; published online September 16, 2014.

Project supported by the Zhejiang Provincial Natural Science Foundation (No. LY12B02017) and the National Basic Research Program of China (973 Program, No. 2011CB710800).

浙江省自然科学基金(No. LY12B02017)、国家重点基础研究发展计划(973 计划, No. 2011CB710800)资助项目. 
泛地应用于有机化学和生物化学领域. 而 PEG 的强亲 水性以及 $\mathrm{AA}$ 的可聚合性决定了它们是合成水凝胶的重 要原料。

水凝胶是一种以水为分散介质且具有网状交联结 构的高分子共聚物. 它的含水量高、生物适应性强, 使 其在最近几年获得生物医学领域的广泛关注 ${ }^{[4 \sim 6]}$. 水凝 胶的合成方法有物理交联法和化学交联法, 其中化学交 联法主要有自由基聚合法、迈克尔加成法、酶催化法、 光引发法和点击反应法, 通常来说水凝胶的合成一般都 采用化学交联法合成.

水凝胶的高聚物骨架本身具有不可降解性, 加上自 由基共聚法在一定程度上也限制了酯基的降解作用，从 而使水凝胶的可降解性较低. 近十年来, 许多学者研究 了各种方法如引入 $\mathrm{SH}$ 、聚乳酸、二茂铁一元酸等来提 高、控制水凝胶的降解能力, 在生物医药领域有重要应 用. 当包埋药物的水凝胶被注射到生物体内, 具有特定 降解速率的水凝胶缓慢持续地向体内释放出药物, 从而 发挥疗效.

水凝胶的合成可以追溯到 1993 年, Hubbell 及其合 作者 ${ }^{[7]}$ 第一次报道了可水解降解的 PEG 与 PLA 或 PGA 的交联共聚物的合成路线. 不仅如此, 他们还预测 PEG 的分子质量、链的长度、聚酯的聚合度等都能影响水凝 胶的降解能力.

2000 年 Metters 等 ${ }^{[8]}$ 构建出了一个数学模型 $\mathbf{1}$, 并指 出可降解的交联聚合物的水解速率、聚合物浓度和交联 密度都能影响 PEG-bis-PLA 共聚物的产率. 在接下来几 年 Mikos、Hubbell 等多位化学家 ${ }^{[9,10]}$ 探索了可水解降解 的水凝胶交联共聚物的合成方法.<smiles>C=CC(=O)OC(C)C(=O)OCCOC(=O)C(C)OC(=O)C=C</smiles>

2006 年 Kinoshita 课题组 ${ }^{[11]}$ 在 $80{ }^{\circ} \mathrm{C}$ 下将 PEG 和 AA 的双酯化物(PEGDA)及其单酯化物(PEGMA), 通过 自由基聚合法, 交联共聚形成水凝胶小球。该水凝胶小 球能够将稀盐酸溶液中的 $\mathrm{AuCl}_{3}$ 还原成 $\mathrm{Au}$. 它的这种吸 金能力来自于 PEG 的水凝胶结构对 $\mathrm{Au}^{3+}$ 具有很强的吸 附作用, 最大可达每克水凝胶吸收 $121 \mathrm{mg}$ 的 $\mathrm{Au}^{3+}$. 更 重要的是, 除 $\mathrm{Au}^{3+}$ 以外的其它重金属离子, 如 $\mathrm{Cu}^{2+}$, $\mathrm{Zn}^{2+}, \mathrm{Fe}^{3+}, \mathrm{Al}^{3+}$ 等, 都不能被该水凝胶小球吸附. 正因 如此, 该水凝胶对 $\mathrm{Au}$ 的选择性还原作用, 能够有效地 将 $\mathrm{Au}$ 从含有多种重金属氯化物的烯酸溶液中提取出来.

2009 年 Guiseppi-Elie 等 ${ }^{[12]}$ 报道以含氨基的丙烯酸 酯为单体和内载二茂铁一元酸的 PEG 衍生物 8 交联, 合 成含氨基丙烯酸酯的聚乙二醇型水凝胶 (Scheme 1). 有
趣的是, 在浸置 $5 \mathrm{~d}$ 后, 由于氨基聚乙二醇型水凝胶的 水解，使二茂铁一元酸能够从水凝胶中分解出来. 正是 由于这种特性推进了水凝胶在合成可控药物载体中的 应用。
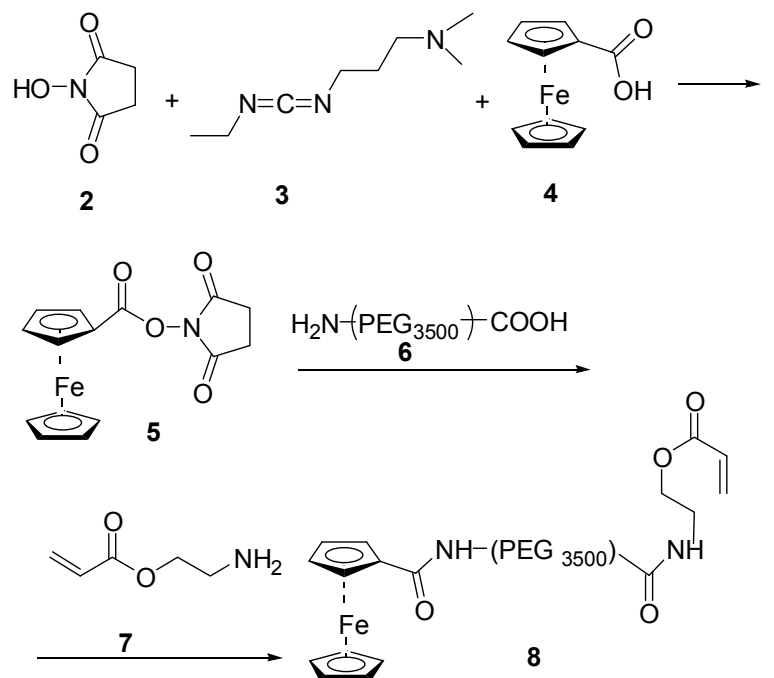

图式 1 Fc-PEG(3500)-AEMA 的合成

Scheme 1 Synthesis of Fc-PEG(3500)-AEMA

与上述自由基聚合法合成凝胶不同，2009 年 Cho 等 ${ }^{[13]}$ 研究出一种新型的两步法, 合成可水解降解的凝 胶(Scheme 2). 该反应在 $50{ }^{\circ} \mathrm{C}$ 下，以 $\mathrm{DMF}$ 作溶剂, 4-甲 氧基苯酚为阻聚剂，将 PEG 转化为 PEGDA，具有较高 的转化率. 此外, 他们以 PEGDA 为单体聚合合成凝胶, 并研究了烷基的链长对水凝胶降解率的影响.
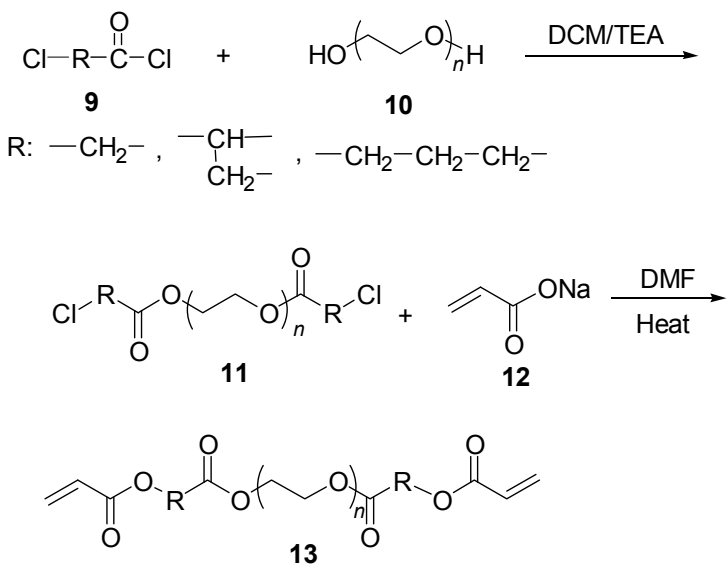

图式 2 聚乙二醇双丙烯酸酯大单体的合成

Scheme 2 Synthesis of PEG-bis-acrylate macromers

为了合成可以同时控制物理特性和化学特性的高 聚物凝胶, 2010 年 Grunlan 等 ${ }^{[14]}$ 采用光交联法, 将 PEGDA 与甲基丙烯酸酯化的聚二甲基硅烷 $\mathrm{PDMS}_{\mathrm{star}}-\mathrm{MA}$ 反应，合成构型独特的无机一有机水凝胶 (Scheme 3). 
<smiles>C=CCOC(=O)C(=C)C</smiles>

图式 3 无机 $\mathrm{PDMS}_{\mathrm{star}}-\mathrm{MA}$ 大单体的合成

Scheme 3 Synthesis of inorganic PDMS star MA macromers

光聚合法是一种合成高分子聚合物的绿色方法, 它 不仅节约能源, 不污染环境而且操作简单, 是现代合成 一些新兴材料的重要方法. 2011 年 West 等 ${ }^{[15]}$ 报道了一 端接有琥珀酸亚氨基的 PEGMA 与肝配蛋白 A1 结合生 成 20, 并通过光交联生成凝胶(Eq. 1). 这提供了一种传 递肝配蛋白 A1 以及合成微血管网状结构的有效方法. 随后, Yui 等 ${ }^{[16]}$ 报道了 PEGDA 与含酰胺类环糊精包合物 的共轭烯酸酯衍生物通过光交联反应生成水凝胶
21.<smiles>C=CC(=O)OCCC(C)C(=O)ON1C(=O)[CH+]CC1=O</smiles>

2011 年 Dijkstra 等 ${ }^{[17]}$ 报道了 PEG-(OH) 8 先后通过氨 化、开环共聚生成三嵌段高分子聚合物 PEG-(PTMC $\left.)_{8}\right)_{-}^{-}$ Acr (Scheme 4). 以此为单体通过光聚合法合成的凝胶 机械性能良好、稳定性强, 在生物医学领域具有良好的 应用前景.

采用光反应原理合成水凝胶时, 产生的放射光容易 导致细胞中毒, 这促使了许多化学家开始研究安全、快 速合成水凝胶的方法. 2012 年 Dijkstra 及其合作者 ${ }^{[18]}$ 通 过迈克尔加成反应, 完成了 PEG- $(\mathrm{OH})_{8}$ 原位接枝聚 $L$-丙 交酯. 他们先在单体末端接上 $\mathrm{SH}$, 再与末端接有烯基 的 PEG 发生迈克尔加成反应来高效快速合成.

同样使用这种方法，还可以将末端接有烯丙酰基的 PEG 与一些天然聚合物, 例如壳聚糖 ${ }^{[19]}$ 、玻尿酸 $\mathbf{2 8}^{[20,21]}$ 和葡聚糖 $\mathbf{2 9}^{[22,23]}$, 原位缩合成网状结构. 通过迈克尔加 成合成的水凝胶可以应用于蛋白质制剂的传递.

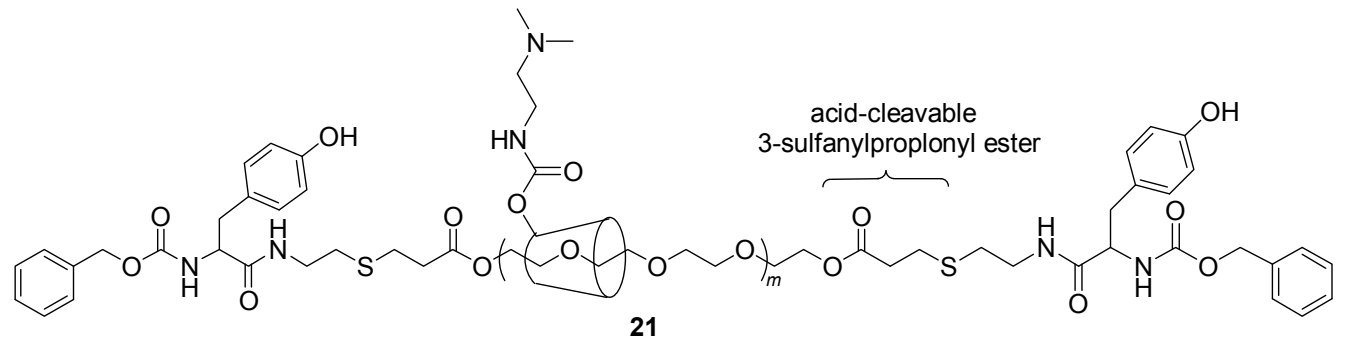

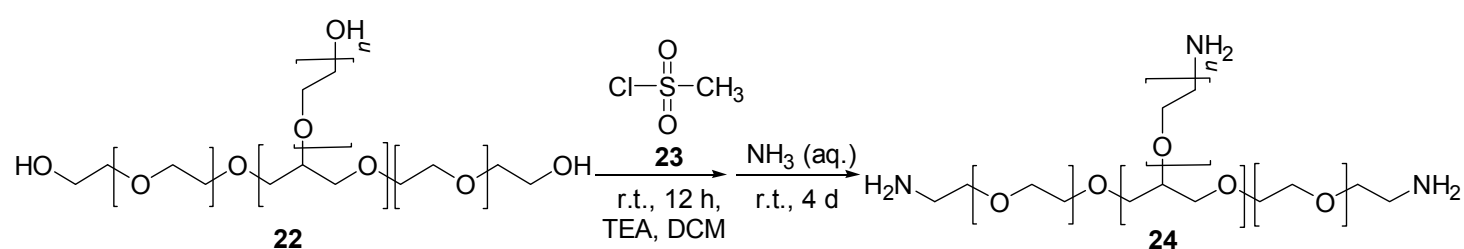

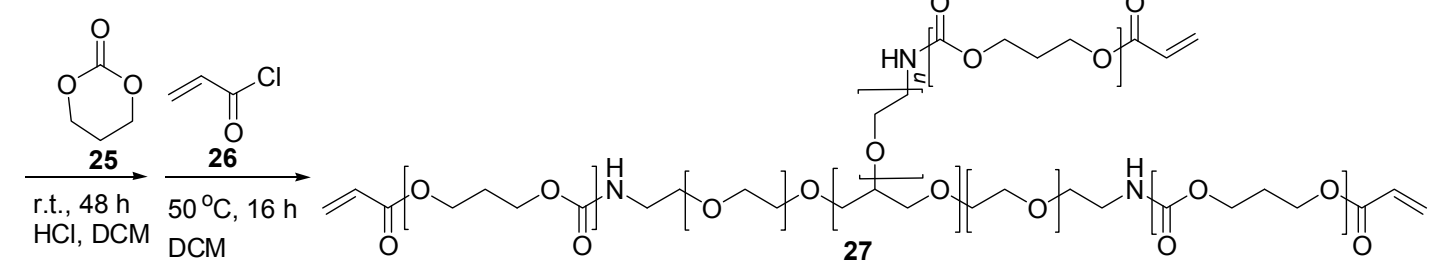

24

图式 4 PEG-(PTMC $)_{8}$-Acr 嵌段共聚物的合成

Scheme 4 Synthesis of PEG-(PTMC $\left.{ }_{9}\right)_{8}$-Acr block copolymers 
<smiles>[Y][R5]#C[Y7]#[Y5]</smiles>

28

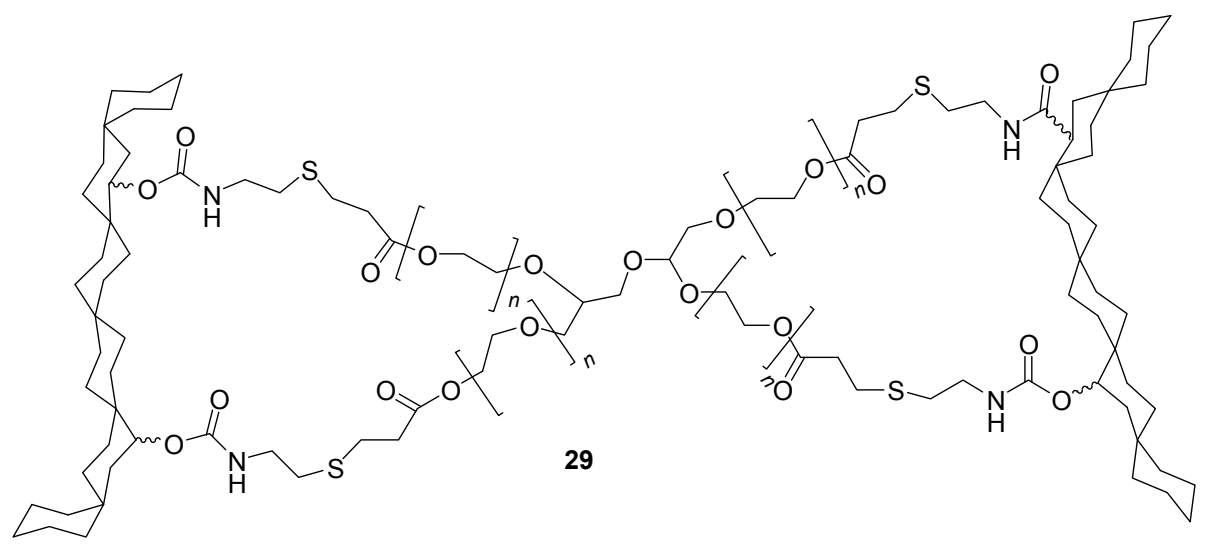

2010 年 $\mathrm{Zhu}$ 等 ${ }^{[24]}$ 以含缩水甘油基甲基丙烯酸酯为 单体和 PEG, 在 $\mathrm{KH}$ 的催化下, 通过质子转移法合成一 种新型的药物载体(Scheme 5). 同年 Xie 等 ${ }^{[25]}$ 研究表明, 支链结构的水凝胶能够促进链之间的作用, 增加交联密 度, 比直链结构的水凝胶具有更大的断裂伸长率和更好 的抗张强度.

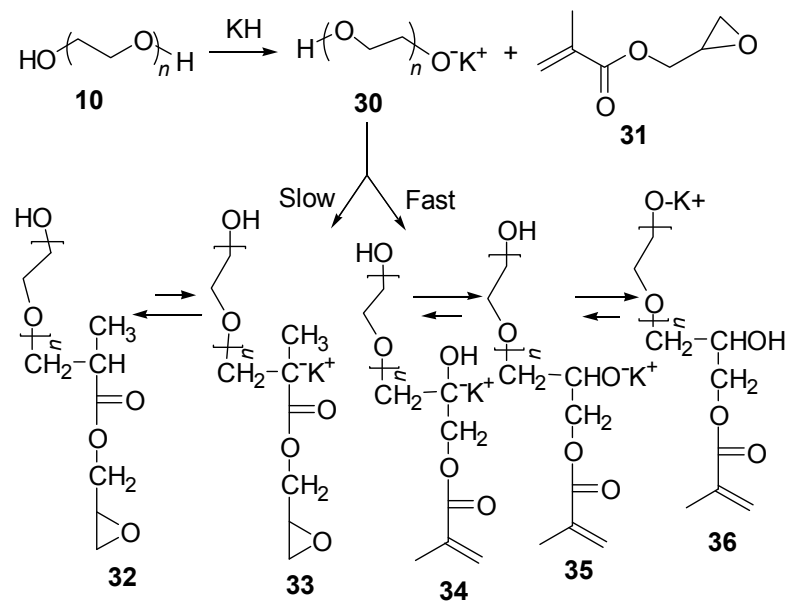

图式 5 PEGGMA 的合成

Scheme 5 Synthesis of PEGGMA

光引发产生的凝胶细胞粘性大, 药物传递的能力有 限. 2013 年 Wang 等 ${ }^{[26]}$ 将 Fenton 体系作为新的引发剂, 在温和的条件下, 通过氧化还原反应产生自由基离子, 来引发交联反应, 合成可注射的水凝胶(Eq. 2). 该水凝 胶还可以与其它生物活性分子结合, 能够将药物快速的
注射到人体内.

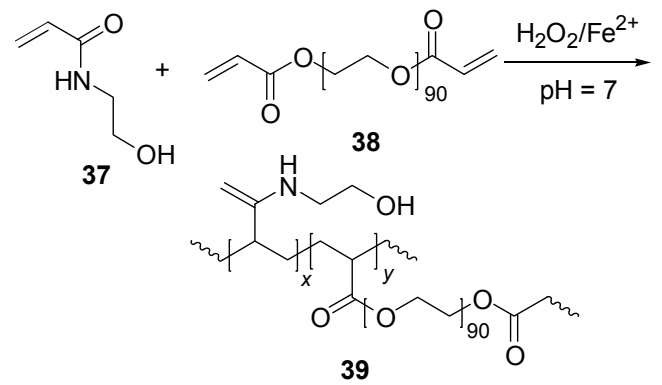

此外, PEG 的化学性质也与链的长度及分子质量有 关. 它的活性随着链长的增长, 分子质量的增加而降低. 因为 PEG 随着链长的增加，容易形成蜷曲状，从而将末 端的羟基包裹在里面，使其不易与反应物接触，降低反 应的活性. 对于分子量大于 2000 的 PEG，通常将它与活 性更高的丙烯酰氯(AC)反应.

早在 1985 年, Brown 等 ${ }^{[27]}$ 就报道了 $\mathrm{PEG}$ 与 $\mathrm{AC}$, 在 三乙胺(TEA) 的催化下反应, 生成 HO-PEG-AC 和 PEG-bis-AC. 报道指出, 只要改变 PEG, AC, TEA 的比 例和反应时间，就能合成不同分子质量的 PEG-bis-AC. 随后 Harris, Bruson, Houben, Katre, Hooper 等 ${ }^{[28 ~ 36]}$ 也分 别报道合成了 PEG-bis-Tos (40), PEG-bis-CN (41), PEG-bis-EtAt (42), PEG-bis-Glu, PEG-bis-TES (43).

2003 年 Jing 等 ${ }^{[37]}$ 报道使用 AC、对甲苯磺酰氯等, 分别与分子量为 2000 和 5000 的 PEG 反应，合成了 PEG 


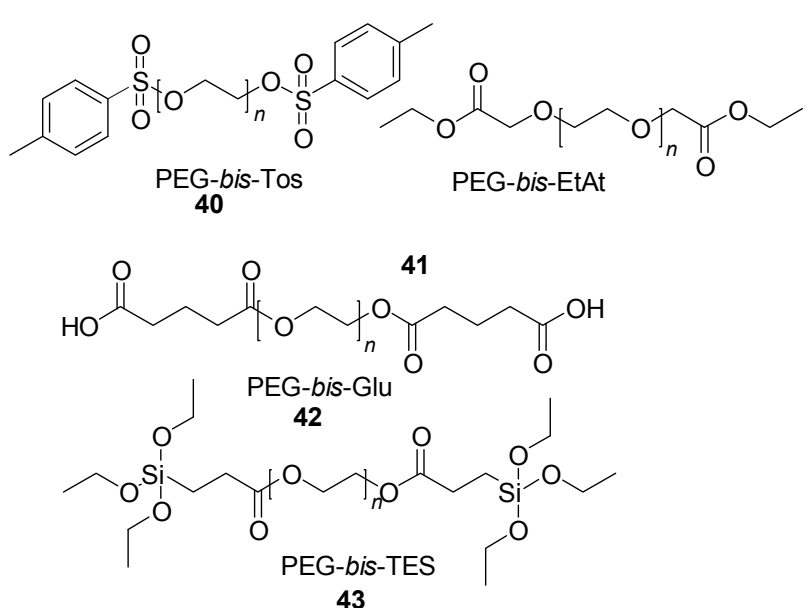

的多种衍生物: CN-PEG-AC (44), Tos-PEG-AC (45), COOH-PEG-AC (46), Glu-PEG-AC (47).

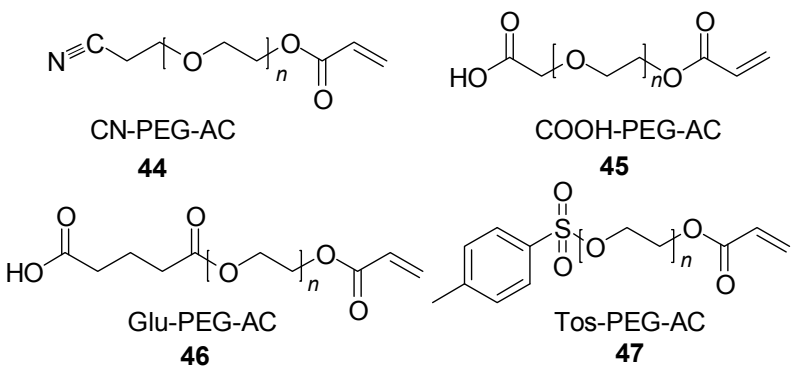

2008 年 $\mathrm{Hu}$ 课题组 ${ }^{[38]}$ 报道了将含多羟基的 PEG 与 PEGDA 单体或丙烯酰胺, 在 UV 光引发剂引发下聚合, 生成凝胶光子晶体(Scheme 6). 这种抗免疫原性, 热灵 敏度高的水凝胶光子晶体的合成为水凝胶开拓了新的 研究与应用领域.

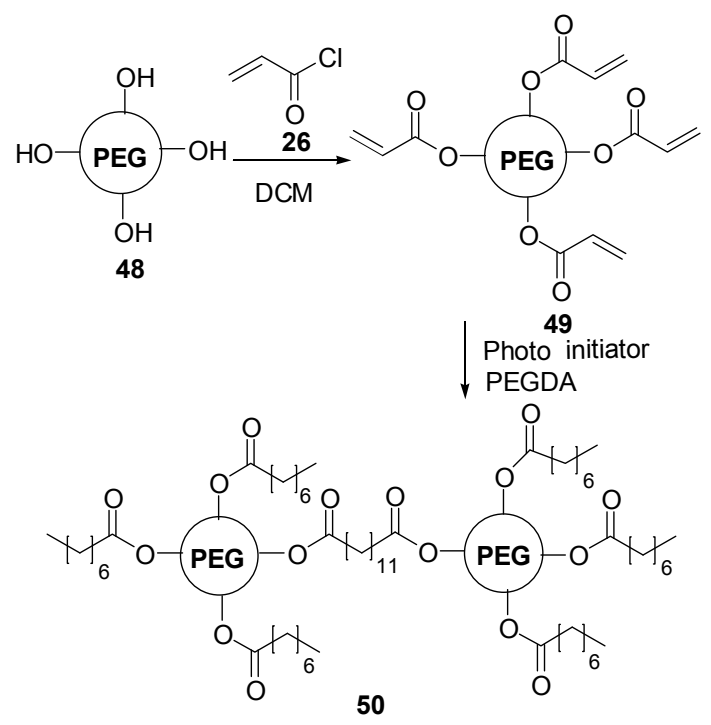

图式 6 PEG 衍生物微凝胶的合成

Scheme 6 Synthesis of PEG derivative microgels
2011 年 $\mathrm{Hsu}$ 等 ${ }^{[39]}$ 实现了 PEG (3400 Da)与 $\mathrm{AC}$ 在 $\mathrm{AgO}$ 和 KI 的催化下选择性合成了 PEGMA (51) (Eq. 3).

$$
\begin{aligned}
& \underset{10}{\mathrm{O}} \mathrm{O}_{n \mathrm{H}}+\mathrm{AgO}+\overbrace{26}^{\mathrm{Cl}} \frac{\mathrm{Kl}}{\substack{\text { Anhydrous } \\
\text { DCM }}} \\
& \overbrace{\mathrm{O}_{51}}^{\mathrm{O}} \mathrm{O}_{\mathrm{O}}^{\mathrm{H}}
\end{aligned}
$$

为了进一步提高凝胶的降解能力, 2012 年 CosgriffHernandez 等 ${ }^{[40]}$ 实现了聚乙二醇双胺与 $\mathrm{AC}$ 反应生成 PEGDAA，并通过光交联形成 PEGDAA 凝胶(Eq. 4). PEGDAA 凝胶比传统的 PEGDA 凝胶具有更强的降解能 力, 其他凝胶特性与 PEGDA 凝胶相近.

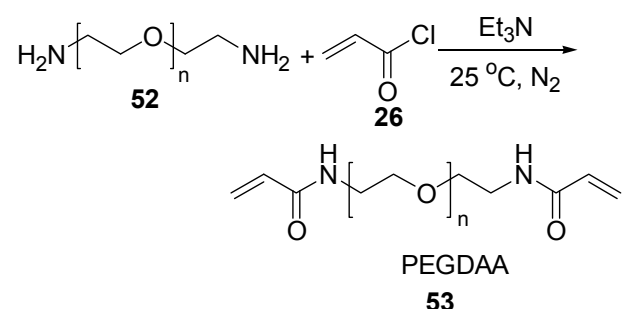

2013 年 Cosgriff-Hernandez 等 ${ }^{[41]}$ 又报道了 PEGDA 先与六亚甲基二异氧酸酯共聚, 形成的聚合物再进一步 与丙烯酰胺聚合, 生成 Aam-PEG-I (54). Aam-PEG-I 是 一种新型的交联剂, 能够对蛋白质进行改性. 此外, Aam-PEG-I 的合成也为蛋白质的功能化开辟了一条新 的道路.

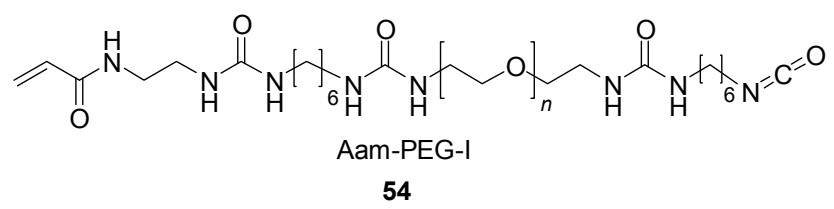

PEG、AA 及其衍生物缩合，除生成水凝胶外，还可 以与其他物质反应生成多种共聚物. 2000 年 Park 及其合 作者 ${ }^{\left[{ }^{[2]}\right]}$ 报道了 PEGMA 在金属 $\mathrm{Na}$ 的催化下, 用丙磺酸 内酯作磺化试剂, 在 $50{ }^{\circ} \mathrm{C}$ 下反应生成磺酸化产物 $\mathbf{5 5}$ (Scheme 7). 55 与丙烯酸十八酯(56)可通过自由基聚合 法共聚生成共聚物 57.

2009 年 Aras 等 ${ }^{[3]}$ 将 MPEGMA 和 2-丙烯酰胺-2-甲 基丙磺酸(APMS)通过水溶液自由基共聚法, 聚合成聚 合物 60 (Eq. 5). 这种聚合物可作为水泥的减水剂, 而且 它的侧链长度和反应时的 $\mathrm{pH}$ 的大小都会影响水泥的流 动性和机械强度.

2011 年 Bianco-Peled 及其合作者 ${ }^{[44]}$ 研究了藻酸硫 醇衍生物 63 和过量的 PEGDA 缩合生成藻酸-聚乙二醇 丙烯酸酯 64 (Scheme 8). 64 的聚合物是一种新型的粘膜 
粘着剂.

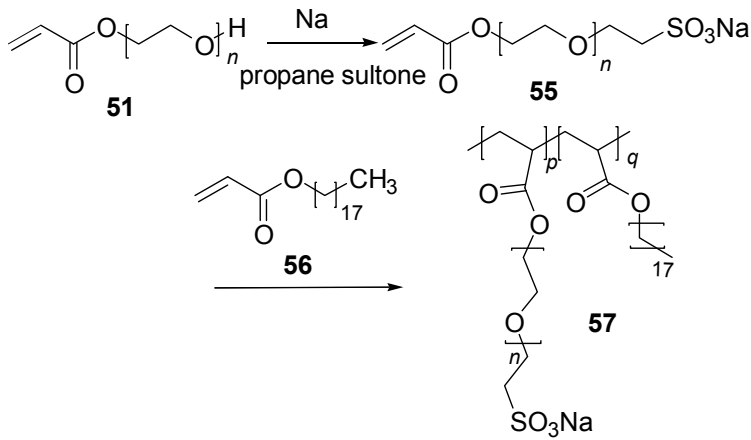

图式 7 PEG-SO 3 共聚物的合成

Scheme 7 Synthesis of PEG-SO $\mathrm{S}_{3}$ copolymers

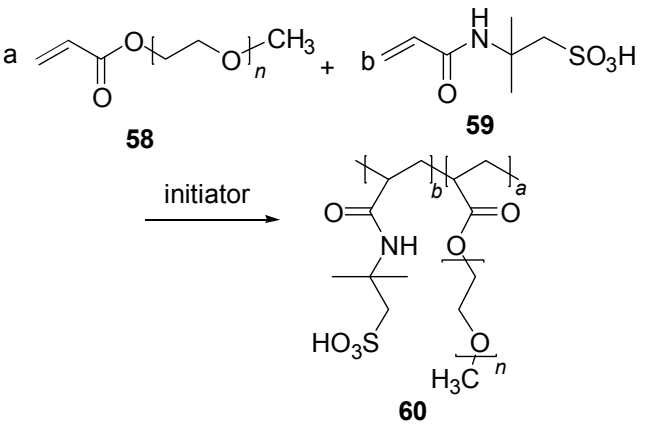

2012 年 Klok 等 ${ }^{[45]}$ 实现了聚乙二醇单甲醚丙烯酸酯 原位接枝肽链. 该物质可以作为一种治疗艾滋病的药物 (Eq. 6).

2013 年 Lei 课题组 ${ }^{[46]}$ 以 PEG 和 AA 为原料, 合成了
聚乙二醇丙烯酸酯的双酯化和单酯化的混合物. 此外, 他们分别用单酯化产物含量高的和双酯化产物含量高 的聚乙二醇丙烯酸酯混合物为原料, 以过硫酸铵为引发 剂, 在 $70{ }^{\circ} \mathrm{C}$ 下反应 $3 \mathrm{~h}$, 分别合成了梳状的 PCE 66 和 桥状的 PCE 67 (Eq. 7).

2014 年 $\mathrm{Liu}$ 等 ${ }^{[47]}$ 报道了聚丙烯酸(PAA)与末端为胺 基的甲氧基聚乙二醇经过脱水缩合形成高聚物 70 (Eq. 8). 该物质可作为水泥减水剂, 并且原料比为 $4: 1$ 时, 表面张力最低, 水泥浆的吸附能力最强, 流动性能最佳.

\section{2 甲基丙烯酸}

甲基丙烯酸(MAA)和 PEG 反应生成可聚合的大分 子单体，将该可聚合大分子单体与其它不饱和酸共聚可 生成聚丙烯酸高效减水剂. 减水剂是水泥混凝土中必不 可少的外加剂，能够有效地改善混凝土的流变性能，提 高混凝土的抗压强度. 传统的减水剂由于掺量大、减水 不够、坞落度损失大等缺点使其应用受到制约, 又由于 发展较早, 各国的研究技术已基本成熟, 发展变化的前 景较小. 聚羧酸系减水剂(PCEs)是继普通减水剂和菜系 减水剂后的第三代高效减水剂, 呈现梳状或刷子状的结 构. PCEs 的主链一般是聚烯酸类, 垂链是由聚乙烯、聚 乙二醇等组成，结构变化多样，可以通过不同的方法合 成不同性能的减水剂, 能够满足建筑工程对水泥混凝土 性能多样化的要求, 具有较好的发展前景. 此外, 聚羧 酸减水剂具有较高的减水率和保坉性, 而且掺量低, 不

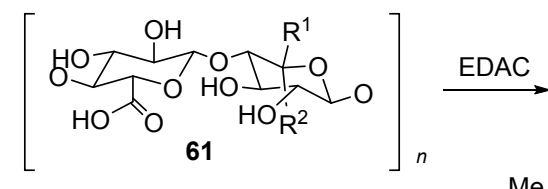

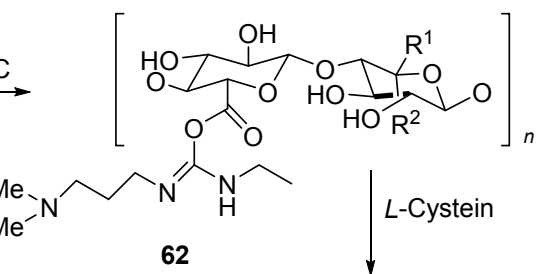

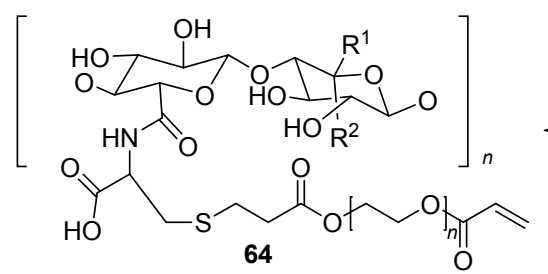
TCEP+PEG-DA

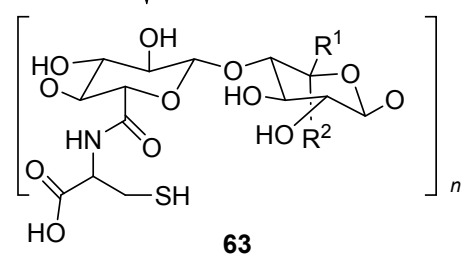

图式 8 Alginate-PEGDA 共聚物的合成

Scheme 8 Synthesis of alginate-PEGDA copolymers

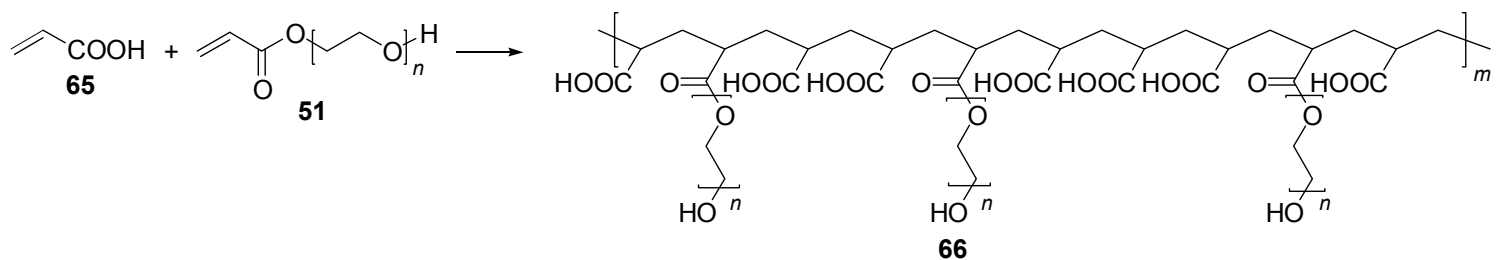




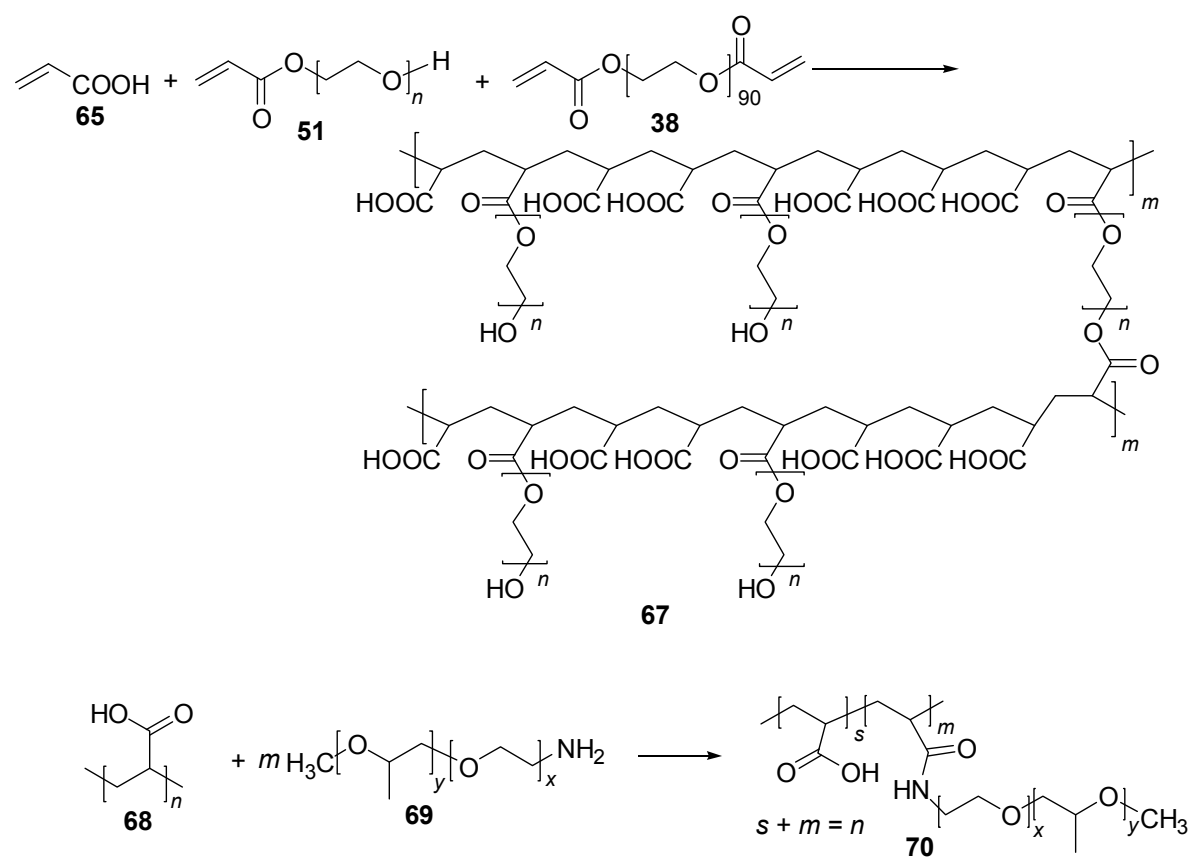

污染环境, 在近几年获得了诸多研究者的关注. 自从 1981 年第一次合成 PCEs 减水剂, 多种 PCEs 的产品陆 续投入工业生产.

MPEG-Type PCEs (71)是市场上第一种减水剂, 由 Nippoh.Shokubai Co. 发明, 被命名为“FC 600” ${ }^{[48]}$. 这种 减水剂可以通过聚甲基丙烯酸主干和甲氧基聚乙二醇 (MPEG)在碱的催化下酯化合成; 也可以由 MAA 与甲氧 基聚乙二醇甲基丙烯酸酯(MPEGMA)通过水溶液自由 基共聚法合成 ${ }^{[49]}$.

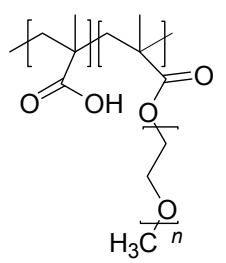

MPEG-type PCEs

71

PAAM-Type PCEs (72) 是投入工业生产的一种两性 减水剂. 它是通过 MAA、MPEGMA 以及含聚酰胺类 (PAAM) 的 PEG 长链共聚合成 ${ }^{[50]}$. 这种减水剂的结构非 常特殊, 它的侧链既含有 PAAM 部分, 又含有 PEO 部 分.

但随着工业的发展, 各国对混凝土的各项性能要求 不断提高, 这就促使了研究者们纷纷投入到合成新型高 效减水剂的研究上来. 2000 年 Yamada 等 ${ }^{[51]}$ 通过分析含 有聚乙二醇长链的梳状聚羧酸系减水剂的化学结构, 研

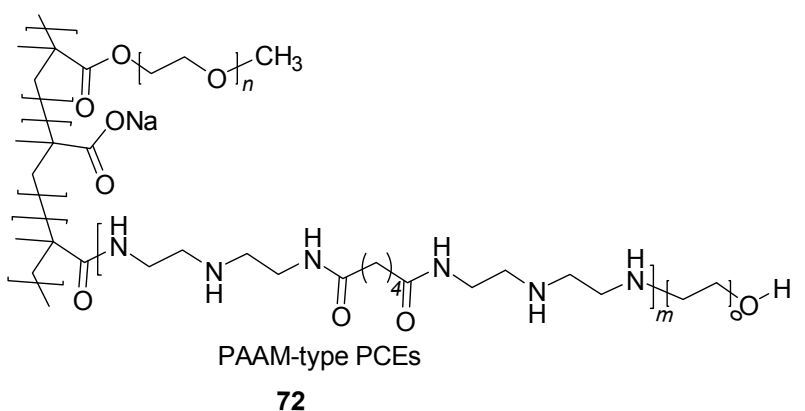

究了水泥颗粒的分散性能. 他们指出，水泥的分散性能 随着聚羧酸系共聚物梳状链(聚乙二醇链)链长的增长, 主链聚合度的增加，磺酸基含量的增加而增大.

2008 年 Felekoglu 课题组 ${ }^{[52]}$ 采用自由基共聚法合成 了 3 种聚羧酸系减水剂, 包含聚羧酸醚类减水剂和聚羒 酸酯类减水剂 $(73 \sim 75)$. 他们分别测试并比较了这 3 种 减水剂对水泥浆的形成时间、工作性能和强度的影响. 研究发现, 虽然聚羧酸醚类减水剂在一定程度上降低了 混凝土的工作性能，但却能更好的保持混凝土的流动
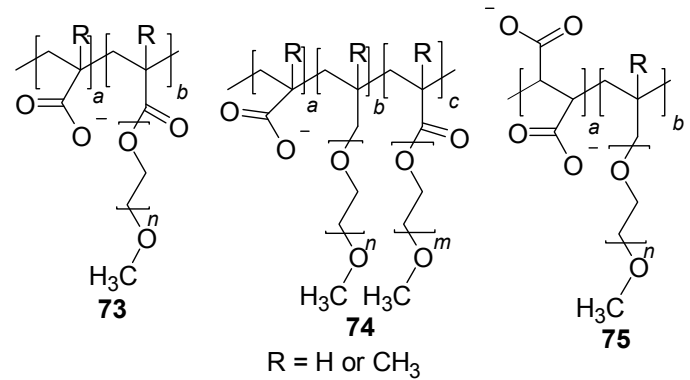
性. 2012 年 $\mathrm{Lv}$ 等 ${ }^{[53]}$ 也合成了不同的聚羧酸醚类和聚羧 酸酯类减水剂, 并得出了类似的结论.

合成聚羧酸减水剂的大单体通常采用甲醇与环氧 乙烷共聚生成 MPEG, 再与 MAA 缩合的两步法合成 (Scheme 9). 2008 年 Plank 等 ${ }^{[54]}$ 报道了环氧乙烷与 MAA 直接一步合成末端含羟基的聚乙二醇甲基丙烯酸酯.

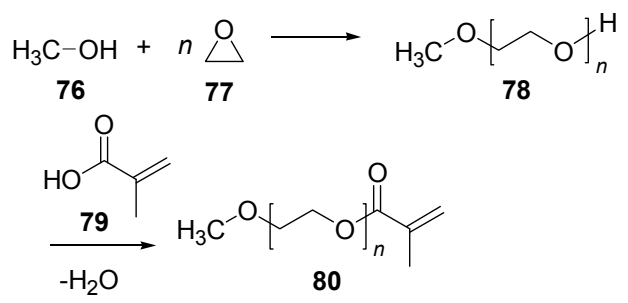

图式 9 MPEG 大单体的合成

Scheme 9 Synthesis of MPEG macromers

2012 年 Plank 等 ${ }^{[55}$ 将 MA 与小分子醇的单甲基丙烯 酸酯共聚，合成了侧链含羟基烷基的减水剂 82 (Eq. 9).

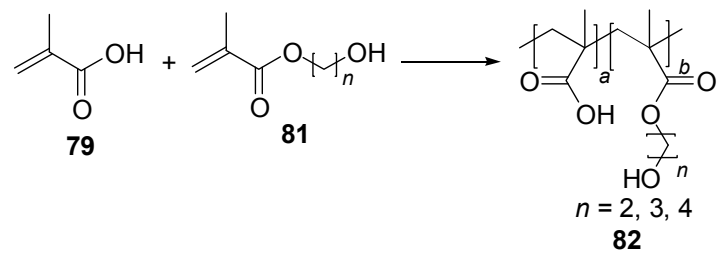

同年 Guo 等 ${ }^{[56]}$ 将马来酸酐与 PEGMMA、甲基丙烯 酰氧基乙基三甲基氯化铵(DMC)共聚, 合成了一种新型 的两性减水剂 83 .

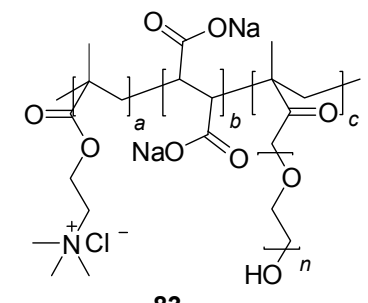

83

近些年来，水泥混凝土朝着绿色、高效、高强度、 低污染以及原料可再生的方向发展. 一些科学家使用天 然高分子材料, 合成出了多种适应性强, 工作性能良好 的绿色水泥减水剂. 事实上, 像多糖 ${ }^{[57]}$ 、淀粉 ${ }^{[58]}$ 、环糊 精 ${ }^{[59 ~ 61]}$ 、酪蛋白 ${ }^{[62]}$ 、纤维素醚类 ${ }^{[63]}$ 等改性的 PCEs 已被 报道出能增加水泥浆的流动性且减水率高. 由于这种天 然高分子材料的可再生性, 使未来这类减水剂的研究具 有广阔的前景.

PEG 与 MAA 及其衍生物反应, 除生成减水剂以外 还可以发生其它多种反应. 2003 年 Anseth 等 ${ }^{[64]}$ 研究了聚 乙二醇甲基丙烯酸酯衍生物 PEG-LA-DM (84)和聚乙烯 醇衍生物 Acr-Ester-PVA (85), 通过光引发共聚合成了
一种可降解的水凝胶.<smiles>C=C(C)C(=O)OCCOC(=O)C(C)C(=O)OCCOC(=O)C(C)OC(=O)C(=C)C</smiles>

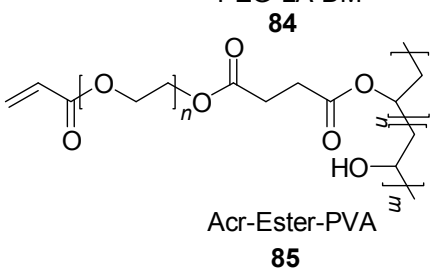

2004 年 Lin-Gibson 等 ${ }^{[65]}$ 将 PEG 与甲基丙烯酸异氰 基乙酯(IEM)缩合，生成内含酰胺的聚乙二醇衍生物 87 (Eq. 10).<smiles>C=C(C)C(=O)CC[N+](=O)[O-]</smiles><smiles>C=C(C)C(=O)OCCNC(=O)OCCOC(=O)NCCOC(=O)C(=C)C</smiles>

2005 年 Lee 等 ${ }^{[66]}$ 以丙交酯、己内酯、磺酰胺甲嘧 啶和 PEG 开环共聚合成三嵌段聚合物 PCLA-PEGPCLA (88). 88 与 OSM 通过自由基聚合，合成一种新型 嵌段共聚物 89 对 $\mathrm{pH}$ 和温度的灵敏性高.

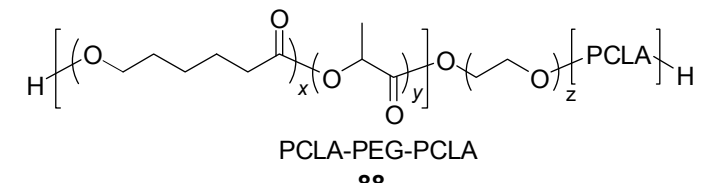

88

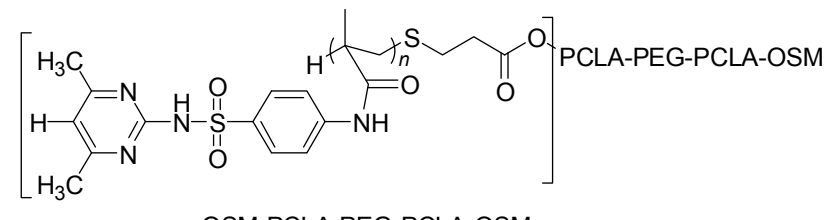

OSM-PCLA-PEG-PCLA-OSM

89

不久 Hennink 等 ${ }^{[67]}$ 通过将 PEG500 的含氮大分子与 $N$-(2-羟基乙基)丙烯酰胺低聚乳酸共聚，合成一种可快 速降解的两性嵌段共聚物 92 (Eq. 11). 此外, Lin-Gibson 课题组 ${ }^{[68]}$ 和 Irvine 课题组 ${ }^{[69]}$ 还报道了将 PEGDA 分别与 聚乳酸、蛋白质等聚合来合成共聚物.

2006 年 Moorhoff 及其合作者 ${ }^{[70]}$ 以对甲苯磺酸为催 化剂，苯作溶剂，合成聚乙二醇氝代甲基丙烯酸酯 97 (Scheme 10). 
<smiles>C=C(C)C(=O)NCCOC(=O)C(C)O</smiles><smiles>C[CH]OCCOC(=O)CCC(C)(C#N)N=NC(C)(C)CCC(=O)OCCO[Ge]</smiles>

$$
91
$$<smiles>CC(C)CCC(=O)OCCO[Ge]</smiles>

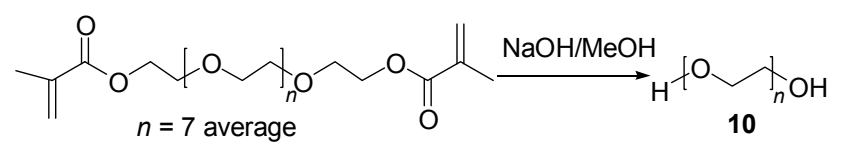

93

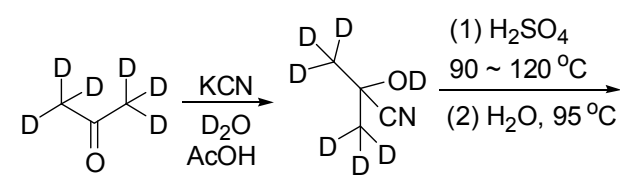

95<smiles>[2H]C([2H])=C(C(=O)O)C([2H])([2H])[2H]</smiles>

96

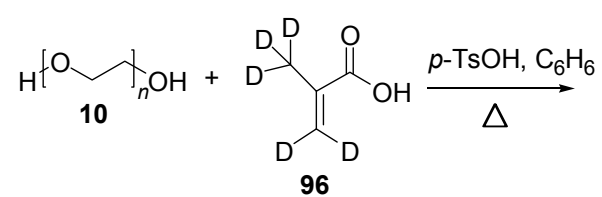<smiles>[2H]C([2H])=C(C(=O)OCCOCC(OCCOC(=O)C(=C([2H])[2H])C([2H])([2H])[2H])C(=O)O)C([2H])[2H]</smiles>

97

图式 10 PEGMA- $d_{5}$ 大单体的合成

Scheme 10 Synthesis of PEGMA- $d_{5}$ macromers

同年 Tosatti 等 ${ }^{[71]}$ 通过聚乙二醇单甲醚甲基丙烯酸 酯与烷基磷酸酯共聚, 合成含有磷酸酯基团的共聚物 98 和 99.
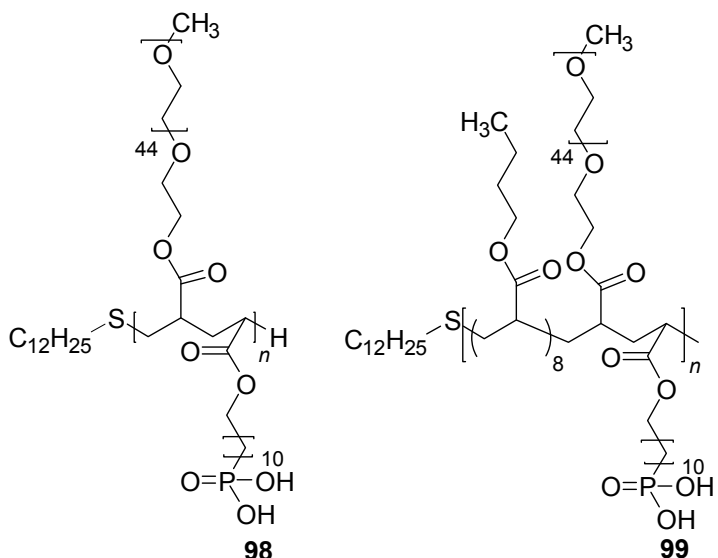

2008 年 Kizhakkedathu 等 ${ }^{[72]}$ 将烷氧戊烷的丙烯酰胺
衍生物 101 与聚乙二醇单甲醚甲基丙烯酸酯，通过 RAFT 聚合，合成含有烷氧戊烷的共聚物 103 (Scheme 11).<smiles>C=CC(=O)NCC1COC(C)(C)O1</smiles>

图式 11 PEGMA- $b$-DDMAA 共聚物的合成

Scheme 11 Synthesis of PEGMA- $b$-DDMAA copolymers

2009 年 $\mathrm{Cai}$ 及其合作者 ${ }^{[73]}$ 首次报道了在温和的可 见光照射下，激发 RAFT 聚合，合成聚乙二醇丙烯酸酯. 这种聚合在 $25{ }^{\circ} \mathrm{C}$ 的水介质中就能实现，而且反应速度 快, 不污染环境. 2011 年 Cao 和 An 课题组 ${ }^{[74]}$ 通过 RAFT 水溶液分散聚合法, 将聚乙二醇甲基丙烯酸酯和二乙二 醇甲基丙烯酸酯合成纳米核一壳结构的水凝胶. 该水凝 胶具有生物活性强、无污染的、热敏性高等特点. 不久 $A n$ 等 $^{[75]}$ 又报道使用亲水的大分子链转移剂，诱发水溶 液分散聚合甲氧基丙烯酸酯衍生物 105, 合成具有生物 活性的纳米微粒 107 (Scheme 12).

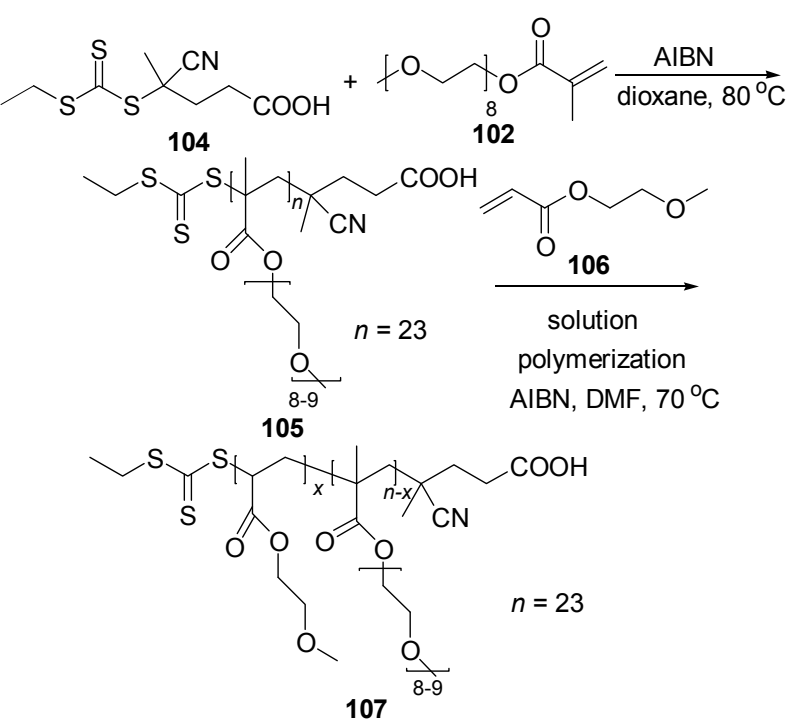

图式 12 PPEGMA- $b$-PMEA 共聚物的合成 Scheme 12 Synthesis of PPEGMA- $b$-PMEA copolymers

2010 年 Hult 等 ${ }^{[76]}$ 报道了不同链长的 PEG、不饱和 酸及硫醇交联生成一种水凝胶涂料. 次年 Brennan 等 ${ }^{[77]}$ 
研究了 PEGDMA 与甲基丙烯酸缩水甘油酯(GMA)、羟 乙基甲基丙烯酸酯共聚, 生成 PEG-co-GMA 和 PEGDMA-co-HEME 水凝胶. 这种水凝胶具有较好的抗 塞性能.

此外 $\mathrm{Xu}$ 及其合作者 ${ }^{[78]}$ 报道合成了新型的可降解的 聚合物半胱氨酸改性的聚乙二醇醋酸聚乳酸酯(Scheme 13).

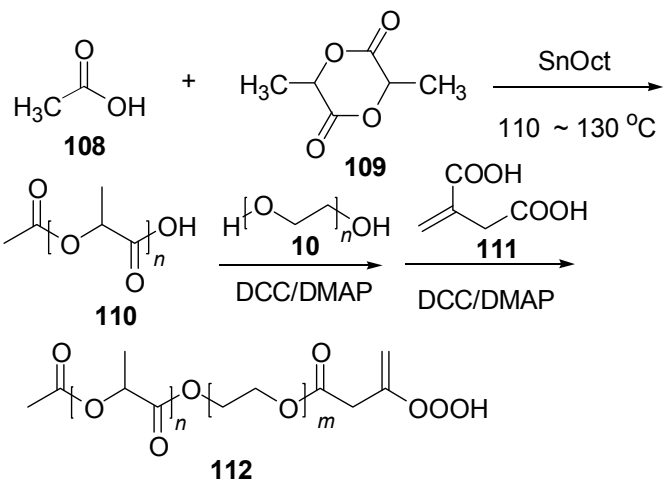

图式 13 半胱氨酸改性的 PEGAc-PLA 的合成

Scheme 13 Synthesis of cysteine modified PEGAc-PLA

同年 Fraser 等 ${ }^{[79]}$ 实现了联吡啶聚乙二醇与甲基丙 烯酸酐酯化形成大分子单体 115. 将 115 与硫酸亚铁作 用，生成含有金属铁离子的 PEG 联吡啶水凝胶 116 (Scheme 14)<smiles>C=C(C)C(=O)OCCOCc1ccnc(-c2cc(COCC(C)O)ccn2)c1</smiles>

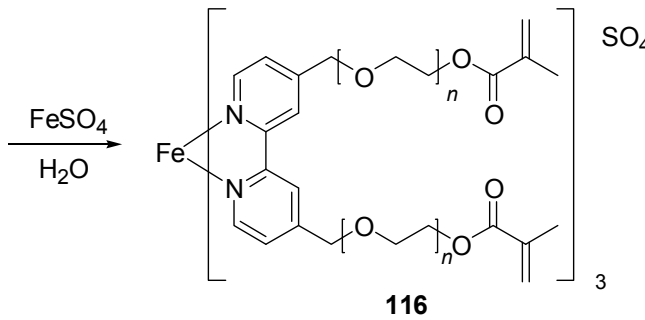

图式 $14\left[\mathrm{Fe}\left\{\text { bpy }(\mathrm{PEG}-\mathrm{MA})_{2}\right\}_{3}\right] \mathrm{SO}_{4}$ 的合成

Scheme 14 Synthesis of $\left[\mathrm{Fe}\left\{\text { bpy }(\mathrm{PEG}-\mathrm{MA})_{2}\right\}_{3}\right] \mathrm{SO}_{4}$

2010 年 Raymo 课题组 ${ }^{[80]}$ 将亲水性的 CdSe-ZnS 核壳纳米颗粒, 沿着聚甲基丙烯酸链接 PEG、伯胺等. 由 于吸收了大分子聚合物或其它活性基团在纳米颗粒的 表面，使该纳米颗粒的性质发生了改变. 研究制备该类 生物活性纳米颗粒, 推进了生物医学领域的纳米苂光探 针技术的研究进展.

随后 Elsner 等 ${ }^{[81]}$ 报道通过电子束触发自由基共聚
接枝聚羧酸酯来引进活性基团.

不久 Müller 及其合作者 ${ }^{[82]}$ 在 $\gamma$ 射线的照射下，选用 4-氧基戊酸二硫代苯甲酸为 RAFT 试剂, 通过 RAFT 聚 合，合成水溶的低聚乙二醇甲基丙烯酸酯. 由于 RAFT 试剂多为非对称的大单体，在聚合的过程中表现得较为 平稳，使得通过 RAFT 聚合得到的复杂聚合物具有均匀 的交联网络结构.

同在 2010 年 Sanyal 等 ${ }^{[83]}$ 将 PEGMMA 与呋喃保护 的马来酰亚胺甲基丙烯酸酯共聚，合成含有共价键的聚 合物. 以此为原料通过一种新型的 Diels-Alder 环加成作 用，与含硫醇的酰胺化合物反应，可生成同时含有马来 酰亚胺和硫醇的活性凝胶。该法实现了在温和的条件 下，将含硫醇的生物素衍生物接到水凝胶上.

2011 年 Agarwal 等 ${ }^{[84]}$ 实现了将聚乙二醇溴代烷基 酯与甲基丙烯酸酯，及含香豆精的甲基丙烯酸酯衍生物 119 共聚, 生成一种抗癌药物 5-氟二嘧啶的载体

120 (Eq. 12)

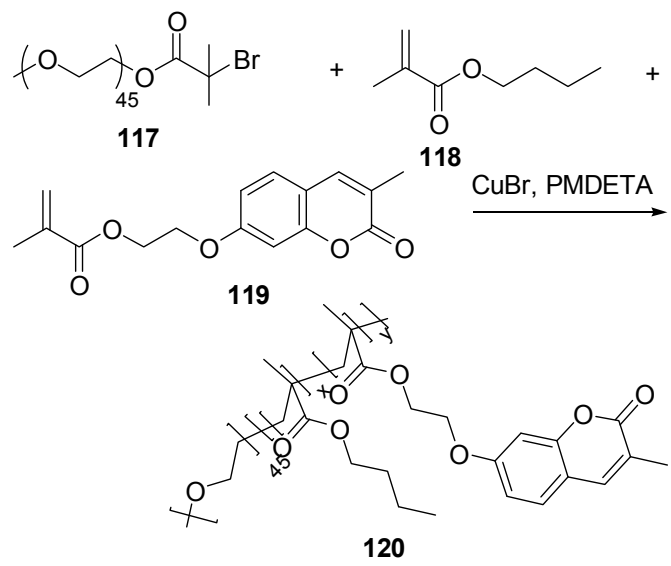

同年Ameringer 等 ${ }^{[85]}$ 报道合成了 PEG 双酯化物接枝 $N$-差基丁二酰亚胺 123 (Scheme 15). 将 123 与聚乙二醇 甲基丙烯酸酯共聚可合成具有特定生物信号的聚合物 涂料.

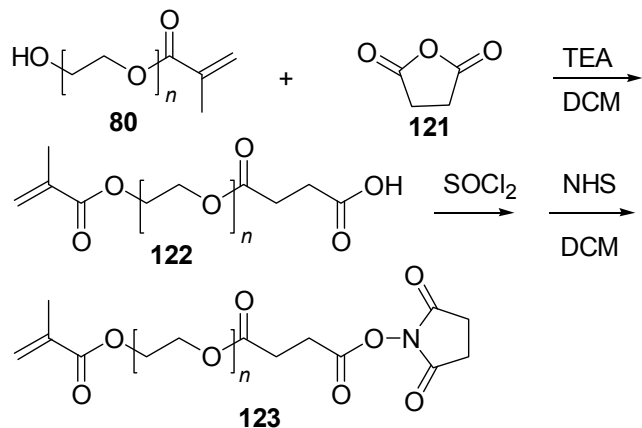

图式 15 含活性 NHS 酯基的 PEGMA 单体的合成 Scheme 15 Synthesis of PEGMAA monomers containing activated NHS ester groups

随后 Miftukhov 等 ${ }^{[86]}$ 以甲苯作溶剂，在 DBU 的催化 
下, 合成了富勒烯取代的甲基丙烯酸酯单体(Scheme 16).

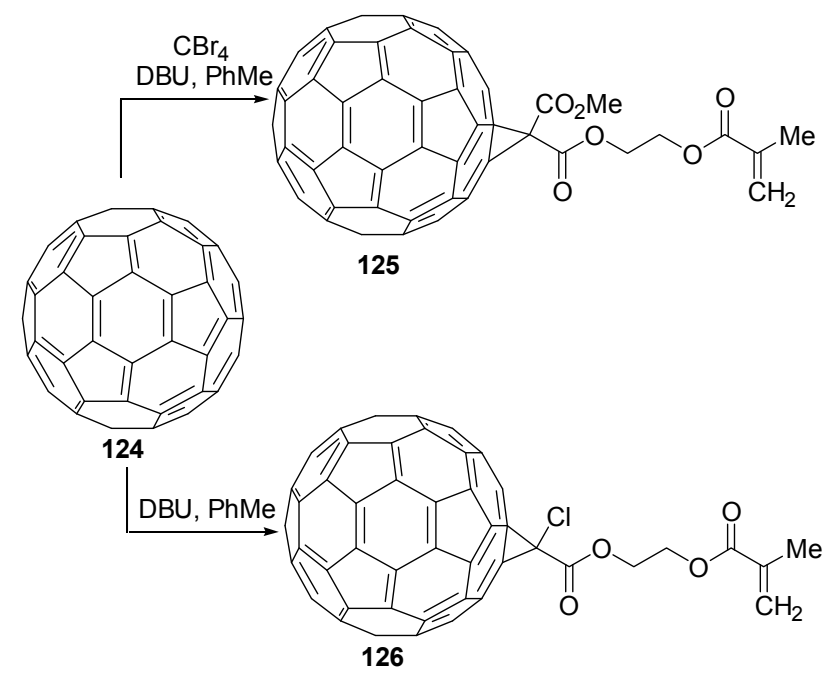

图式 16 含甲基丙烯酸酯富勒烯的合成

Scheme 16 Synthesis of fullerene-containing methacrylates

最近 Najafi 等 ${ }^{[87]}$ 合成了聚羧酸系分散剂涂料(Eq. 13). 他们以甲基丙烯酸和聚乙二醇单甲醚为原料, 合 成新型的水溶性聚羧酸酯共聚物 (mPEGMA-co-PMAA). 这种共聚物可作为理想的涂料分散剂. 经测定, 它不仅 可以维持颗粒的大小, 而且可以增强吸收树脂的性能和 薄膜硬度.

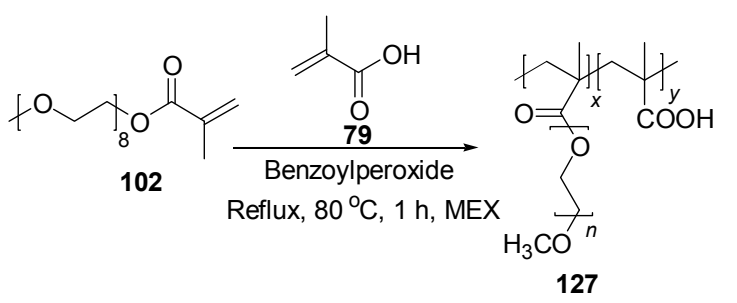

此外, Zhao 等 ${ }^{[88]}$ 以 PEG、MAA 的溴代衍生物先与 含二硫键的烯酸酯共聚, 再与 1-萠丁酸酯化, 获得含有 二硫键和萠基的两性嵌段共聚物.

2013 年 Ma 及其课题组 ${ }^{[89]}$ 选用 PEG 烯基衍生物和 MA、丙烯酰胺为原料, 合成了一种有前景的、高效的 水煤浆添加剂 130 (Eq. 14). 事实证明, 把悬浮的煤溶入 水中形成的水煤浆, 是一种重要的燃料油的替代品. 这 就需要加入高效的外加剂来实现, 而这种聚羧酸外加剂 具有强的空间位阻, 可以使水煤浆具有较好的流动性和 稳定性.

同年 Kasko 等 ${ }^{[90]}$ 实现了聚乙二醇甲基丙烯酸酯衍 生物和多种芳香类化合物的反应，合成茮基位接有醇、 胺、卤素、酸、酯等不同活性基团高聚物 131. 这类物 质在药物的传递上有广泛的作用.

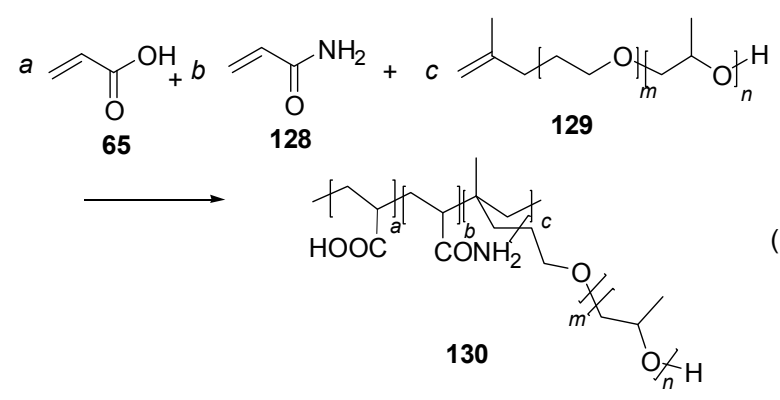

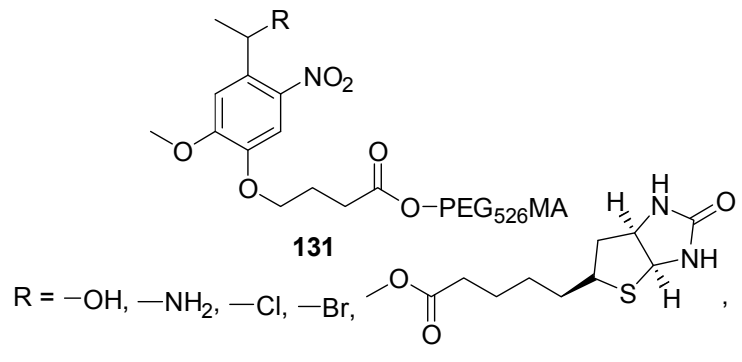<smiles>C=CC(=O)OC</smiles><smiles>COCCC(=O)OCCC(=O)ON1C(=O)CCC1=O</smiles>

不久 Zhang 等 ${ }^{[91]}$ 通过聚乙二醇单甲基烯丙醚与含 乙缩醛功能化的甲基丙烯酸酯共聚, 生成梳状的两性共 聚物 P(mPEGMA-co-TTMA) (Eq. 15). 此外, Ye 等 ${ }^{[92]}$ 将 PEG 先后与烷基苯甲酰氯、甲基丙烯酰氯发生缩合反应, 也合成了一种新型的两性树形一线团大单体.

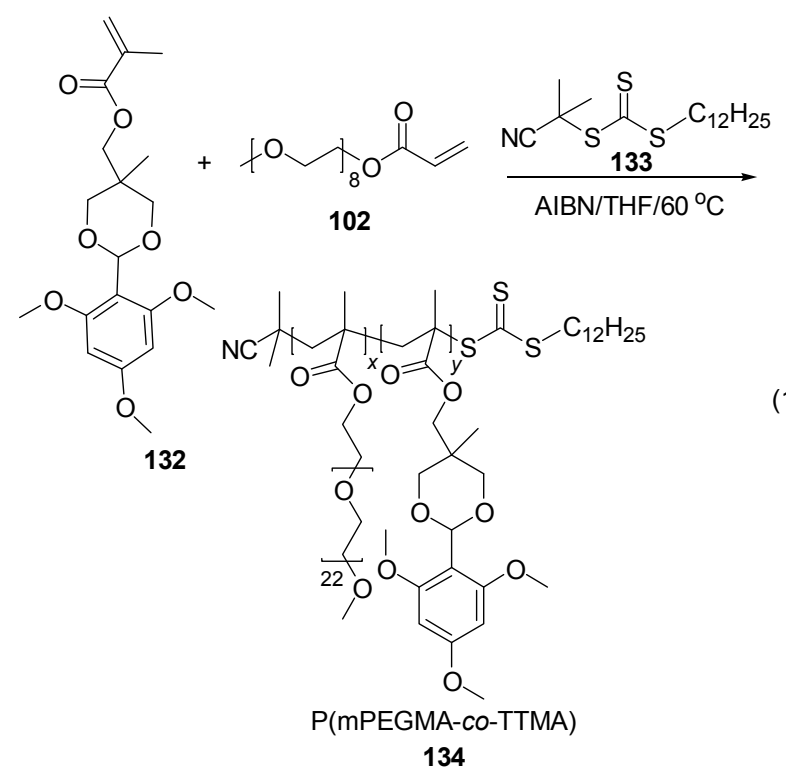

2014 年 $\mathrm{Li}$ 等 ${ }^{[93]}$ 将聚乙二醇单甲基丙烯酸酯与含有 氨基和放基的半胱氨酸开环聚合, 形成双嵌段共聚物, 
并表明该物质在药物传递系统中有重要的应用.

\section{3 马来酸}

马来酸䣶 $(\mathrm{MAH})$ 与 $\mathrm{PEG}$ 反应可生成马来酸单聚乙 二醇单甲醚活性单体，是除聚乙二醇甲基丙烯酸酯外， 合成聚羧酸混泥土减水剂大单体的另一种有效合成方 法. 减水剂在混凝土中的作用受到多种因素的影响, 如 溶解度、空间位阻以及与其他外加剂的相容性等. 乙烯 类共聚物减水剂在近几年发展迅速, 但也存在着容易分 离水、与缓凝剂的相容性差等缺点. 相比于 MAA, MAH 不仅在酯化的过程中, 具有不易自聚、较难氧化等特点, 而且生成的减水剂的空间位阻更大, 水溶性更佳, 适应 性更强. 此外, MAH 和 PEG 在聚合的过程中接枝其它 单体, 可生成许多化工产品中的重要中间体, 使其具有 较好的应用前景.

APEG-Type PCEs 和 HPEG-Type PCEs 这两种减水 剂产品就是通过马来酸䣶分别与甲氧基聚乙二醇烯丙 醚、甲氧基聚乙二醇甲基烯丙醚共聚合成 ${ }^{[94,95]}$.

2010 年 Khalfallah 及其合作者 ${ }^{[96]}$ 报道了以甲苯作溶 剂, 在对甲苯磺酸的催化下, 合成马来酸冠醚 136, 并 将其与含氟烷基化合物反应, 生成氟化环聚乙二醇琥珀 酸酯 138 (Scheme 17). 其中, 马来酸冠醚的产率与 PEG 的聚合度有关, PEG 的聚合度越大, 马来酸冠醚的产率 越低.

不久 $\mathrm{Lu}$ 等 ${ }^{[97]}$ 用 $\mathrm{MAH}$ 与 $\mathrm{PEG}$ 缩合后与柠檬酸缩合 生成聚乙二醇马来酸柠檬酸酯 PEGMC, 将 PEGMC 与 聚乙二醇丙烯酸(PEGAA)、AA、烯丙基磺酸钠(SAS)以 及 MAA 共聚, 生成新型的烯基接枝双聚型减水剂大单 体(Eq. 16). 当原料的物质的量比 PEGAA：PEGMC： $\mathrm{AA}: \mathrm{SAS}: \mathrm{MAA}=0.5: 0.1: 0.2: 0.05: 0.03$ 时, 减水 剂的各项性能最佳.

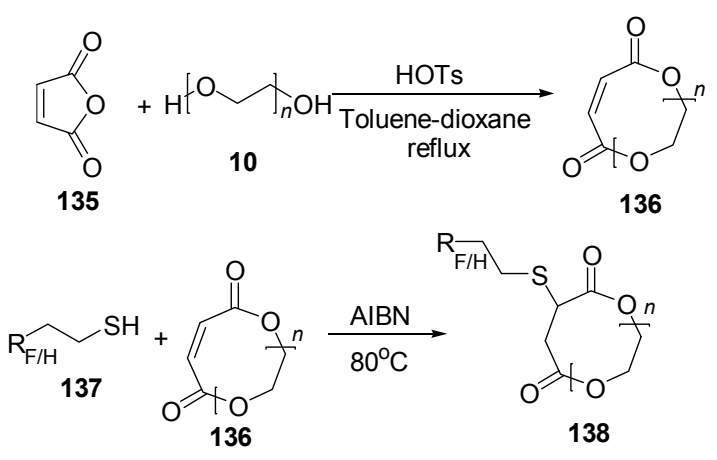

图式 17 马来酸冠醚的合成

Scheme 17 Synthesis of Maleic acid crown ethers

2010 年 $\mathrm{Li}$ 等 ${ }^{[98]}$ 首次在水溶液中, 通过 Diels-Alder 环加成合成基于 $N$-乙烯基-2-吡咯烷酮水凝胶. 其中, 亲

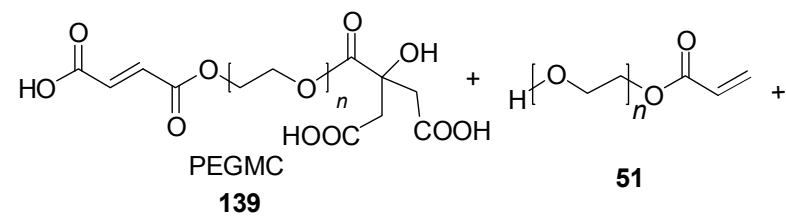<smiles>C=C[C+]=CC(=O)O</smiles>

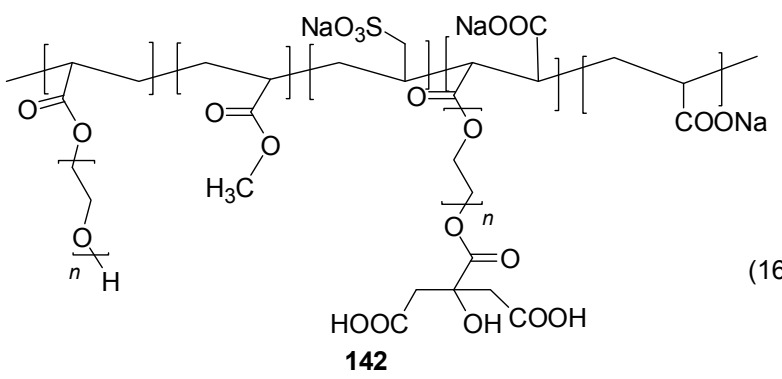

二烯体高聚物 145 是由马来酰亚胺的羧化物 144 与 $\mathrm{PEG}$ 在脱水剂 $N, N^{\prime}$-二环己基碳二亚胺的作用下合成的 (Scheme 18).

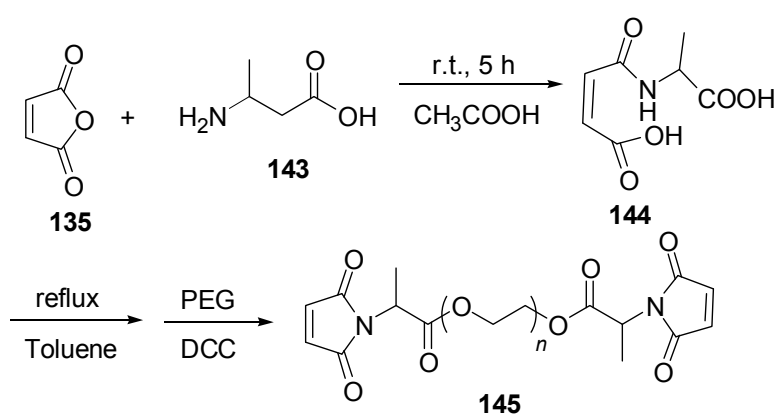

图式 18 聚乙二醇亲二烯体羧化物的合成

Scheme 18 Synthesis of PEG dienophile carboxylates

不久 Huang 及其合作者 ${ }^{[99]}$ 在对甲苯磺酸的催化下， 通过开环加成合成 PEG 和 MAA 的共聚物 146, 并用它 改性消旋聚乳酸(Eq. 17). 经过聚乙二醇马来酸酐共聚 物改性的消旋聚乳酸, 具有柔软性并且降解速率高.

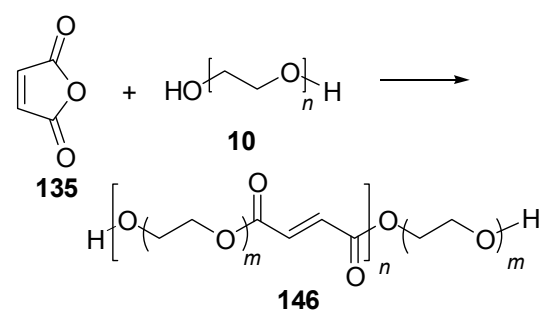

2012 年 $\mathrm{LV}$ 课题组 ${ }^{[100]}$ 合成了一种 $\beta$-环糊精改性的 新型聚羧酸系减水剂 149 (Scheme 19). 他们先以 DMF 为溶剂, 在 $80{ }^{\circ} \mathrm{C}$ 下搅拌 $10 \mathrm{~h}$, 合成 $\beta$-环糊精马来酸酯 单体 148. 将其与 MA、甲基烯丙基磺酸、PEGMMA 共 
聚生成减水剂 149. 由于 $\beta$-环糊精的空间位阻和引气作 用，使水泥浆具有较好的分散性和流动性.

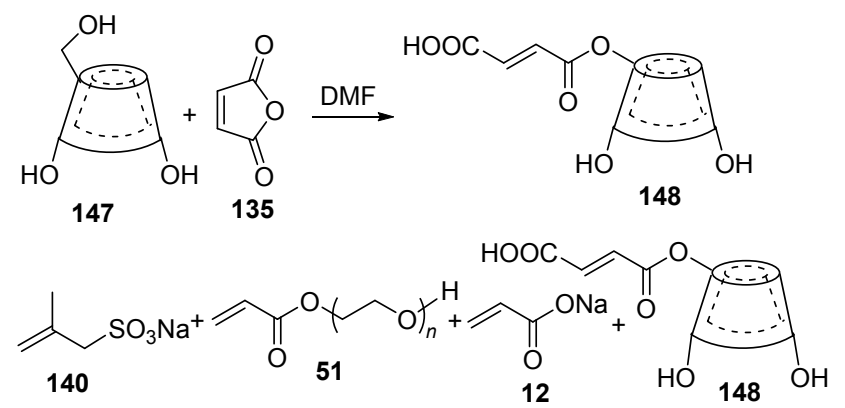

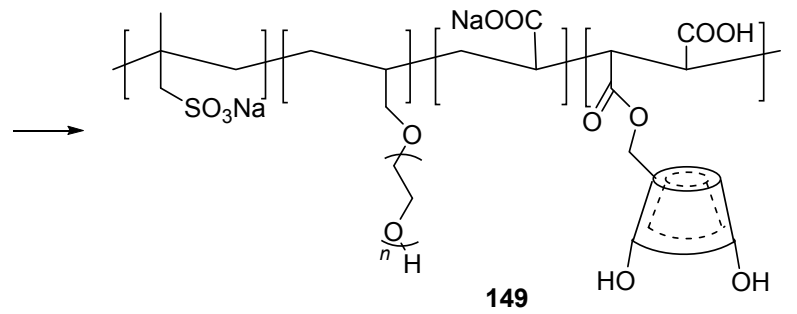

图式 $19 \beta$-环糊精改性的聚羧酸系高效减水剂的合成

Scheme 19 Synthesis of $\beta$-CD modified superplasticizers

2012 年 Guo 等 ${ }^{[101]}$ 将马来酸酐与 PEGMMA、甲基 丙烯酰氧基乙基三甲基氯化铵(DMC)共聚, 合成了一种 新型的两性 PCE.

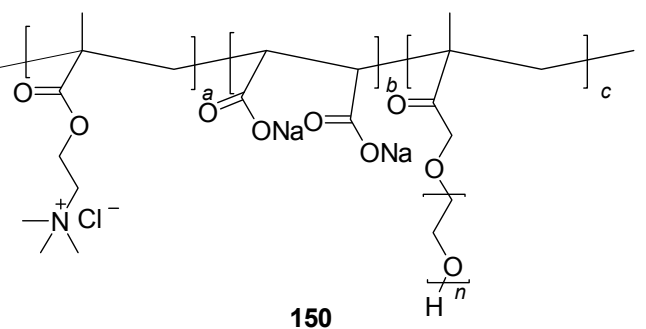

2013 年 $\mathrm{LV}$ 等 ${ }^{[102]}$ 将聚乙二醇单乙烯基醚分别与 MAH、亚甲基丁二酸共聚合成 MPCs (151), IPCs (152) 两种类型的聚羧酸醚类减水剂. 这两种减水剂由于连接 主链上的羒基个数不同, 导致混凝土的流动性、密实性 和抗压强度等受到影响.
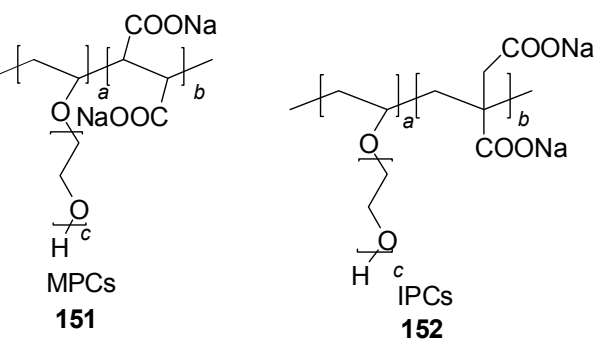

2013 年 Plank 等 ${ }^{[103]}$ 报道使用 MAH 及其衍生物和
MPEGA 聚合, 合成了含有五元环的新型减水剂 154 (Eq. 18). 这种减水剂的阴离子浓度高, 空间位阻小, 能 够更密实的覆盖在水泥表面, 在硫酸盐水泥中比传统水 泥具有更好的工作性能.

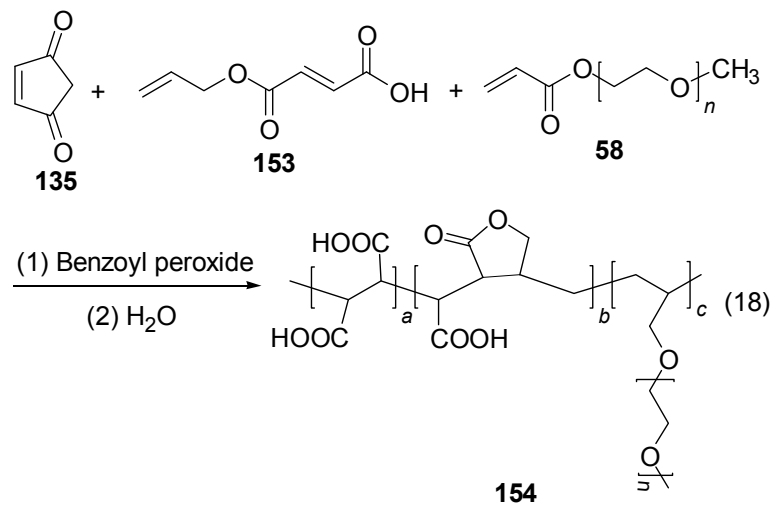

\section{4 含芳环的共轭烯酸}

近几年来含芳环的共轭烯酸与 PEG 的缩合反应也 受到了许多研究者的关注. 2002 年 Yang 课题组 ${ }^{[104]}$ 报道 了对羟基苯甲酸与 PEG 在 DMF 中缩合生成 157. 将所 得的 157 与硫氰酸铵在二氯甲烷中反应, 获得改性的聚 乙二醇硫脲衍生物 159 (Scheme 20). 用这种方法获得的 聚乙二醇硫嫝衍生物反应条件温和、纯度较高而且产率 高达 90\%.
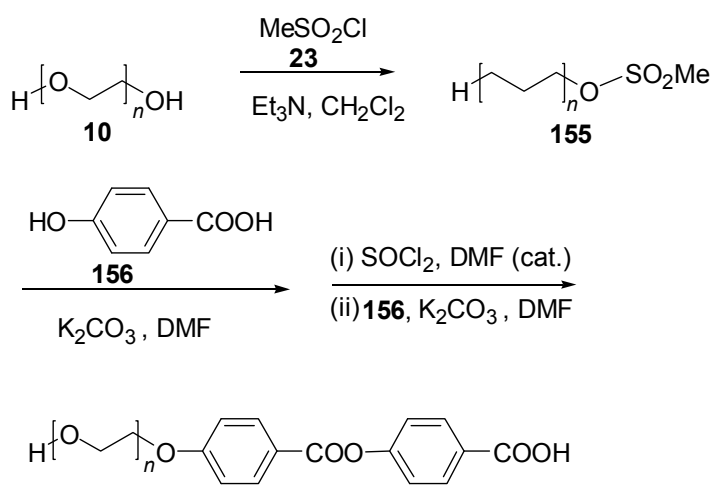

157

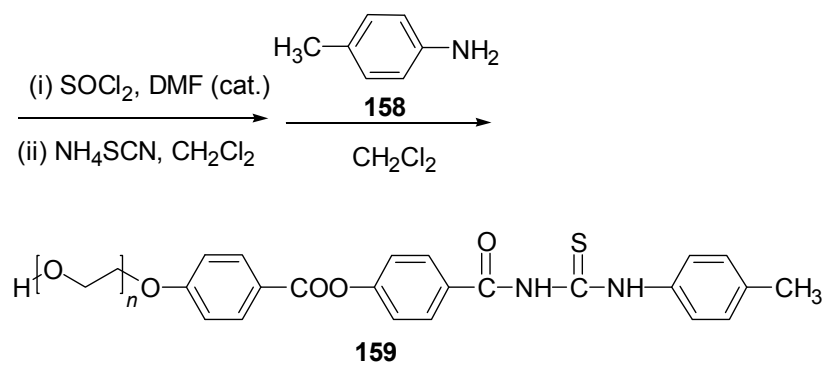

图式 20 硫嫝衍生物的合成

Scheme 20 Synthesis of thiourea derivatives

2002 年 Chandrasekhar 及其合作者 ${ }^{[105]}$ 在三氟甲烷磺 
酸钪的催化下, 在甲苯中回流 $18 \mathrm{~h}$, 合成一系列苯甲酸 与 $\mathrm{PEG}$ 的聚合物(Eq. 19).

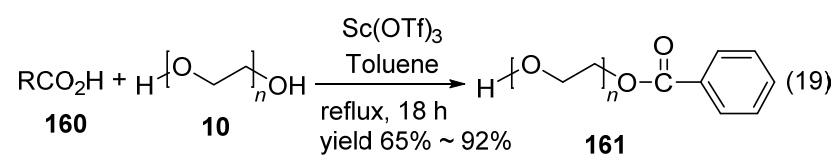<smiles>[R]OC(C)=O</smiles>

2004 年 Chen 等 ${ }^{[106]}$ 以对苯二甲酸为原料合成新型 的苯甲酰基氟化试剂 163 (Scheme 21). 它与 PEG 的共 聚物可作为制备 2-取代苯并咪唑、2-取代苯并塞唑或 2取代苯并噁唑的桥梁.

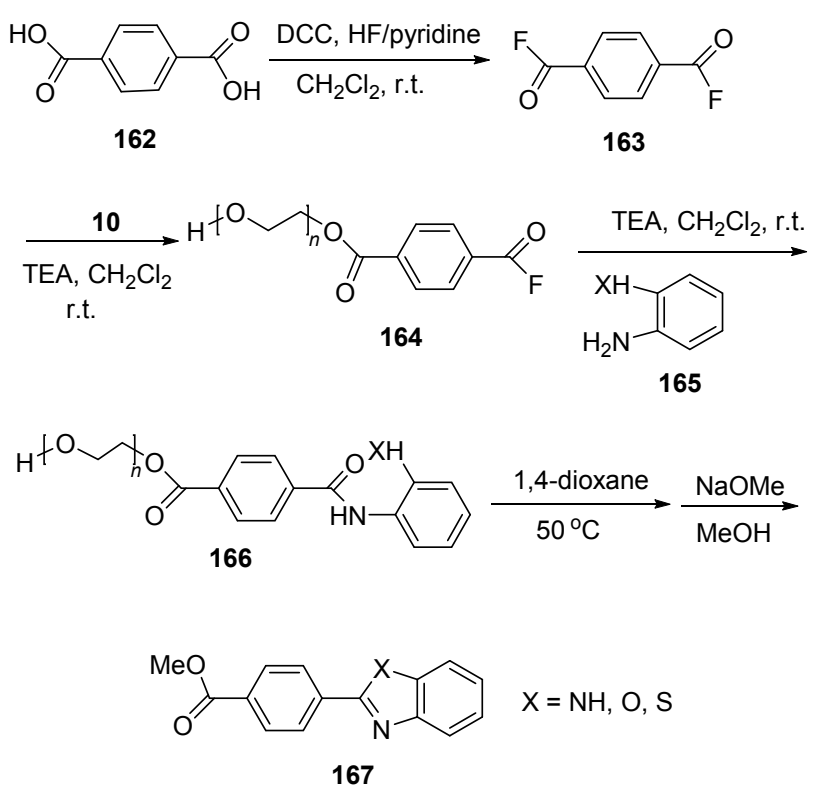

图式 21 2-取代苯并咪唑、2-取代苯并噻唑和 2-取代苯并噁 唑的合成

Scheme 21 Synthesis of 2-substituted benzimidazoles, benzoxazoles and benzothiazoles

2011 年 Wang 等 ${ }^{[107]}$ 报道将三种具有不同链段性能 的聚合物, 即作为柔性段链的 PEG、作为脂肪族单元的 聚对苯二甲酸丁二酯和作为刚性芳香链段的聚乳酸, 共 聚合成一种生物降解能力强的新型高分子聚合物材料.

2011 年 Yang 等 ${ }^{[108]}$ 通过超声雾化法, 原位表面聚合 聚乙二醇苯甲醛与乙二醇壳聚糖, 合成复合的微凝胶胶 囊.

2012 年 Evans 及其合作者 ${ }^{[109]}$ 以含芳环的丙烯酸酯 衍生物和 PEG 反应，合成化合物 169 (Scheme 22). 以此 为原料, 通过和其它不饱和化合物 RAFT 聚合生成一系 列可光降解的水凝胶.

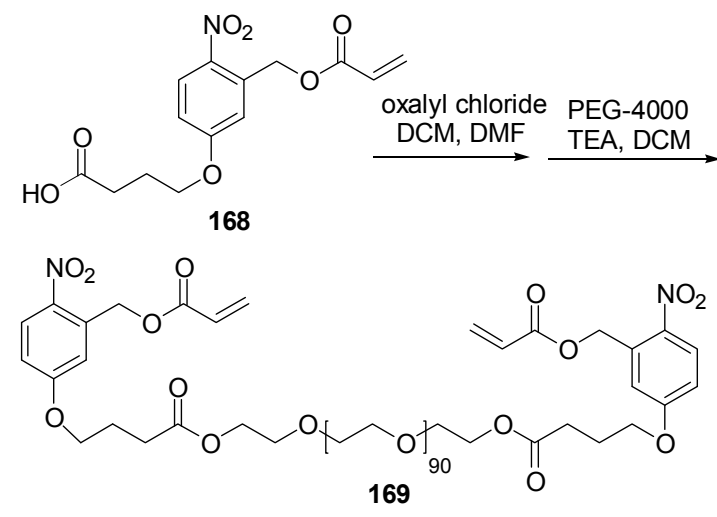

图式 22 聚乙二醇芳环衍生的合成

Scheme 22 Synthesis of PEG derivatives-containing aromatic ring

\section{5 结论与展望}

本文介绍了不同的共轭烯酸与 PEG 缩合反应, 及 其在有机化学和生物化学中的应用. 长链不饱和羧酸酯 聚合物在水凝胶、涂料、胶粘剂、表面活性剂、水泥减 水剂等领域得到了广泛的应用. 由于不同结构和组成的 聚合物具有不同的物理和化学性能，其在实际应用中范 围和深度也不一样. 绿色、高效、高性能、原料可再生 的聚合物是一个重要的研究方向，使用天然高分子材料 合成的聚合物正符合了这种要求。随着对不饱和羧酸酯 单体的不断深入研究, 相信高性能羧酸酯聚合物在化工 生产过程中会占有越来越重要的地位，并向着功能多样 化、生态化、国际化方向发展.

\section{References}

[1] Winnefeld, F.; Becker, S.; Pakusch, J.; Gotz, T. Cem. Concr. Res. 2007, 29, 251.

[2] Zhang, A. F.; Du, X. D.; Lei, J. H.; Liu, Y.; Bi, Y. Anal. Lett. 2010 , 43,858

[3] Lu, S. H.; Liu, G.; Ma, Y. F.; Li, F. J. Appl. Polym. Sci. 2010, 117, 273.

[4] Peppas, N. A.; Bures, P.; Leobandung, W.; Ichikawa, H. Eur. J. Pharm. Biopharm. 2000, 50, 27.

[5] Drury, J. L.; Mooney, D. J. Biomaterials 2003, 24, 4337.

[6] Hennink, W. E.; Nostrum, C. F. Adv. Drug Delivery Rev. 2002, 54, 13.

[7] Sawhney, A. S.; Pathak, C. P.; Hubbell, J. A. Macromolecules 1993, 26, 581

[8] Metters, A. T.; Bowman, C. N.; Anseth, K. S. J. Phys. Chem. B 2000, 104, 7043 .

[9] Jo, S.; Shin, H.; Shung, A. K.; Fisher, J. P.; Mikos, A. G. Macromolecules 2001, 34, 2839.

[10] Metters, A.; Hubbell, J. Biomacromolecules 2005, 6, 290.

[11] Kinoshita, T.; Ishigaki, Y.; Nakano, K.; Yamguchi, K.; Akita, S.; Nii, S.; Kawaizumi, F. Sep. Purif. Technol. 2006, 49, 253.

[12] Boztas, A. O.; Guiseppi-Elie, A. Biomacromolecules 2009, 10, 2135.

[13] Cho, E.; Kutty, J. K.; Datar, K.; Lee, J. S.; Vyavahare, N. R.; 
Webb, K. J. Biomed. Mater. Res. 2009, 90A, 1073.

[14] Hou, Y.; Schoener, C. A.; Regan, K. R.; Munoz-Pinto, D.; Hahn, M. S.; Grunlan, M. A. Biomaromolecules 2010, 11, 648.

[15] Saik, J. E.; Gould, D. J.; Keswani, A. H.; Dockinson, M. E.; West, J. L. Biomaromolecules 2011, 12, 2715.

[16] Tamura, A.; Yui, N. J. Mater. Chem. B 2013, 1, 3535.

[17] Buwalda, S. J.; Perez, L. B.; Teixeira, S.; Calucci, L.; Forte, C.; Feijen, J.; Dijkstra, P. J. Biomaromolecules 2011, 12, 2746.

[18] Buwalda, S. J.; Dijkstra, P. J.; Feijen, J. Macromol. Chem. Phys. 2012, 213, 766.

[19] Teng, D. Y.; Wu, Z. M.; Zhang, X. G.; Wang, Y. X.; Zheng, C.; Wang, Z.; Li, C. X. Polymer 2010, 51, 639.

[20] Censi, R.; Fieten, P. J.; Martino, W. E.; Hennink, W. E.; Vormonden, T. Macromolecules 2010, 43, 5771.

[21] Jin, R.; Teixeira, L. S. M.; Kouwels, A.; Dijkstra, P. J.; Van Blitterswijk, C. A.; Karperjien, M.; Feijen, J. Acta Biomater. 2010, $6,1968$.

[22] Hiemstra, C.; Van der Aa, L. J.; Zhong, Z. Y.; Dijkstra, P. J.; Feijen, J. Biomaromolecules 2007, 8, 1548.

[23] Hiemstra, C.; Vander Aa, L. J.; Zhong, Z. Y.; Dijkstra, P. J.; Feijen, J. Macromolecules 2007, 40, 1165.

[24] Pang, Y.; Liu, J.; Wu, J.; Li, G.; Wang, R.; Su, Y.; He, P.; Zhu, X.; Yan, D.; Zhu, B. Bioconjugate Chem. 2010, 21, 2073.

[25] Yang, J.; Gong, C.; Shi, F. K.; Xie, X. M. J. Phys. Chem. B 2012, 116, 12038.

[26] Sun, L.; Zhang, S.; Zhang, J.; Wang, N.; Liu, W.; Wang, W. J. Mater. Chem. B 2013, 1, 3932.

[27] Brown, R. D.; Champion, R.; Elmes, P. S.; Godfrey, P. D. J. Am. Chem. Soc. 1985, 107, 4109.

[28] Harris, J. M.; Dust, J. M.; Sedaghat-Herati, M. R.; McGill, R. A.; Upton, C. Am. Chem. Soc., Polym. Prepr. 1989, 30, 356.

[29] Kao, W. J.; Lee, D. Biomaterials 2001, 22, 2901.

[30] Bruson, H. A. Org. React. 1949, 5, 79.

[31] Houben-Weyl, H.; Muller, E.; Verlag, T. Methoden Org. Chem. 1970, XIII, 377.

[32] Katre, N. V.; Knauf, M. J.; Laird, W. J. Proc. Natl. Acad. Sci. U. S. A. 1987, 84, 1487.

[33] Delgado, C.; Francis, G. E.; Fisher, D. Crit. Rev. Ther. Drug Carrier Syst. 1992, 9, 249.

[34] Hooper, R.; Lyons, L. J.; Moline, D. A.; West, R. Organometallics 1999, $18,3249$.

[35] Hempenius, M. A.; Lammertink, R. G. H.; Vancso, G. J. Macromolecules 1997, 30, 266.

[36] Nixon, P. G.; Winter, R.; Castne, D. G.; Holcomb, N. R.; Grainger, D. W.; Gard, G. L. Chem. Mater. 2000, 12, 3108.

[37] Jing, L.; Kao, W. J. Biomaromolecules 2003, 4, 1055.

[38] Cai, T.; Wang, G.; Thompson, S.; Marquez, M.; Hu, Z. B. Macromolecules 2008, 41, 9508.

[39] Hsu, C.; Olabisi, R. M.; Olmsted-Davis, E. A.; Davis, A. R.; West, J. L. J. Biomed. Mater. Res. 2011, 98, 53.

[40] Browning, M. B.; Cosgriff-Hernandez, E. Biomaromolecules 2012, 13, 779 .

[41] Browning, M. B.; Rusell, B.; Rivera, J.; Hook, M.; Cosgriff-Hernandez, E. M. Biomaromolecules 2013, 14, 2225.

[42] Lee, H. J.; Park, K. D.; Park, H. D.; Lee, W. K.; Han, D. K.; Kim, S. H.; Kim, Y. H. Colloids Surf. B 2000, 18, 355.

[43] Büyükyagcī, A.; Tuzcu, G.; Aras, L. Cem. Concr. Res. 2009, 39, 629.

[44] Davidovich-Pinhas, M.; Bianco-Peled, H. Acta Biomater. 2011, 7, 625.

[45] Danial, M.; Dulmen, Tim H. H.; Aleksandrowicz, J.; Potgens, Andy, J. G.; Klok, H. A. Bioconjugate Chem. 2012, 23, 1648.
[46] Lei, J. H.; Li, H.; Du, X. D.; Zhang, A. F. Iran Polym. J. 2013, 22, 117.

[47] Liu, X.; Wang, Z.; Zhu, J.; Zheng, Y.; Cui, S.; Lan, M.; Li, H. Colloids Surf. A 2014, 448, 119.

[48] Sakai, E.; Ishida, A.; Ohta, A. J. Adv. Concr. Technol. 2006, 4, 211.

[49] Plank, J.; Pollmann, K.; Zouaoui, N.; Andres, P. R.; Schaefer, C. Cem. Concr. Res. 2008, 38, 1210.

[50] Amaya, T.; Ikeda, A.; Imamura, J.; Konayashi, A.; Saito, K.; Danzinger, W.; Tomoyose, T. WO 2000039045, 2000 [Chem. Abstr. 2000, 133, 93537].

[51] Yamada, K.; Takahashi, T.; Hanehara, S.; Matsuhisa, M. Cem. Concr. Res. 2000, 30. 197.

[52] Felekoglu, B.; Sarikahya, H. Cons. Buil. Mater. 2008, 22, 1972.

[53] Lv, S.; Duan, J.; Gao, R.; Cao, Q.; Li, D. Polym. Adv. Technol. 2012, 23, 1596.

[54] Plank, J.; Pollmann, K.; Zouaoui, N.; Andres, P. R.; Schaefer, C. Cem. Concr. Res. 2008, 38, 1210.

[55] Lei, L.; Plank, J. Cem. Concr. Res. 2012, 42, 1299.

[56] Guo, W.; Sun, N.; Qin, J.; Zhang, J. Pei, M.; Wang, Y.; Wang, S. J. Appl. Polym. Sci. 2012, 125, 283.

[57] Peschard, A.; Govin, A.; Pourchez, J.; Fredon, E.; Bertrand, L.; Maximilien, S.; Guihot, B. J. Eur. Ceram. Soc. 2006, 26, 1439.

[58] Izaguirre, A.; Lanas, J.; Alvarez, J. I. Carbohydr. Polym. 2010, 80, 222.

[59] Lv, S. H.; Gao, R. J.; Duan, J. P.; Li, D.; Cao, Q. J. Appl. Polym. Sci. 2012, 125, 396.

[60] Lv, S.; Gao, R.; Cao, Q.; Li, D.; Duan, J. Cem. Concr. Res. 2012, $42,1356$.

[61] Li, Y.; Guo, H.; Hang, Y.; Zheng, J.; Li, Z.; Yang, C.; Lu, M. Carbohydr. Polym. 2014, 102, 278.

[62] Bian, H.; Plank, J. Cem. Concr. Res. 2013, 51, 1.

[63] Patural, L.; Marchal, P.; Govin, A.; Grosseau, P.; Ruot, B.; Deves, O. Cem. Concr. Res. 2011, 41, 46.

[64] Martens, P. T.; Bryant, S. J.; Anseth, K. S. Biomaromolecules 2003, 4, 283.

[65] Lin-Gibson, S.; Bencherif, S.; Cooper, J. A.; Wetzel, S. J.; Antonucci, J. M.; Vogel, B. M.; Horkay, K.; Washburn, N. R. Biomaromolecules 2004, 5, 1280.

[66] Shim, W. S.; Yoo, J. S.; Bae, Y. H., Lee, D. S. Biomaromolecules 2005, 6, 2930.

[67] Rijcken, C. J. F.; Veldhuis, T. F. J.; Ramzi, A.; Meeldijk, J. D.; Nostrum, C. F.; Hennink, W. E. Biomaromolecules 2005, 6, 2343.

[68] Zhang, K.; Simon, C. G. Washburn, N. R.; Antonucci, J. M.; Lin-Gibson, S. Biomaromolecules 2005, 6, 1615.

[69] Jain, S.; Yap, W. T.; Irvine, D. J. Biomaromolecules 2005, 6, 2590.

[70] Moorhoff, C. M.; Cook, W. D. Monatsh. Chem. 2006, 137, 449.

[71] Zoulalian, V.; Monge, S.; Zurcher, S.; Textor, M.; Robin, J. J.; Tosatti, S. J. Phys. Chem. B 2006, 110, 25603.

[72] Rossi, N. A. A.; Zou, Y. Q.; Scott, M. D.; Kizhakkedathu, J. N. Macromolecules 2008, 41, 5272.

[73] Shi, Y.; Gao, H.; Lu, L.; Cai, Y. L. Chem. Commun. 2009, 60, 1368.

[74] Shen, W. Q.; Chang, Y. L.; Liu, G. Y.; Wang, H. F.; Cao, A. N.; An, Z. S. Macromolecules 2011, 44, 2524.

[75] Liu, G. Y.; Qiu, Q.; Shen, W. Q.; An, Z. S. Macromolecules 2011, 44, 5237.

[76] Lundberg, P.; Bruin, A.; Klijnstra, J. W.; Nystrom, A. M.; Johansson, M.; Malkoch, M.; Hult, A. Am. Chem. Soc., Appl. Mater. Interfaces 2010, 2, 903.

[77] Magin, C. M.; Finlay, J. A.; Clay, G.; Callow, M. E.; Callow, J. A.; Brennan, A. B. Biomaromolecules 2011, 12, 915.

[78] Xu, B.; Yuan, J. F.; Wang, Z. F.; Gao, Q. Y. J. Microencapsulation 
2009, 26, 659 .

[79] Fiore, G. L.; Klinkenberg, J. L.; Pfister, A.; Fraser, C. L. Biomaromolecules 2009, 10, 128.

[80] Yildiz, I.; Deniz, E.; McCaughan, B.; Cruickshank, S. F.; Callan, J. F.; Raymo, F. M. Langmuir 2010, 26, 11503.

[81] Elsner, C.; Ernst, C.; Buchmeiser, M. R. J. Appl. Polym. Sci. 2011, 119, 1450.

[82] Millard, P. E.; Barner, L.; Reinhardt, J.; Buchmeiser, M. R.; Barner-Kowollik, C.; Müller, A. H. E. Polymer 2010, 51, 4319.

[83] Kosif, I.; Park, E. J.; Sanyal, R.; Sanyal, A. Macromolecules 2010, 43, 4140.

[84] Jin, Q.; Mitschang, F.; Agarwal, S. Biomacromolecules 2011, 12, 3684.

[85] Ameringer, T.; Fransen, P.; Bean, P.; Johnson, G.; Pereira, S.; Evans, R. A.; Thissen, H.; Meagher, L. J. Biomed. Mater. Res. 2012, $100 \mathrm{~A}, 370$.

[86] Torosyam, S. A.; Biglova, Y. N.; Mikheev, V. V.; Khlitova, Z. T.; Gimalova, F. A.; Miftukhov, M. S. Mendeleev Commun. 2012, 22, 199.

[87] Najafi, F.; Ranjbar, Z.; Shirkavand, H., B.; Montazeri, S. J. Appl. Polym. Sci. 2012, 126, 877.

[88] Yuan, L.; Liu, J. C.; Wen, J. G.; Zhao, H. Y. Langmuir 2012, 28, 11232.

[89] Ma, S.; Zhao, P.; Guo, Y.; Zhong, L.; Wang, Y. Fuel 2013, 111, 648.

[90] Griffm, D. R.; Schlosser, J. L.; Lam, S. F.; Nguyen, T. H.; Mayhard, H. D.; Kasko, A. M. Biomaromolecules 2013, 14, 1199.

[91] Zhao, J. Q.; Wang, H. Y.; Liu, J. J.; Deng, L. D.; Liu, J. F.; Dong, A. J.; Zhang, J. H. Biomaromolecules 2013, 14, 3973.

[92] Ye, H. H.; Ren, B. Y.; Liu, R.; Peng; J. Tong, Z. Ind. Eng. Chem. Res. 2013, 52, 11858.

[93] Fu, X. H.; Ma, Y. N.; Shen, Y.; Fu, W. X.; Li, Z. B. Biomaromolecules 2014, 15, 1055.
[94] Akimoto, S.; Honda, S.; Yasukohchi, T. EP 0291073, 1988 [Chem. Abstr. 1989, 110, 120207].

[95] Hamada, D.; Yamato, F.; Mizunuma, T.; Ichikawa, H. DE 10048139, 2001 [Chem. Abstr. 2001, 134, 284753].

[96] Khalfallah, A.; Ayari, A.; Boughariou, B.; Hedhli, A. J. Inclusion Phenom. Macrocyclic Chem. 2010, 67, 1.

[97] Lu, S. H.; Liu, G.; Ma, Y. F.; Li, F. J. Appl. Polym. Sci. 2010, 117, 273.

[98] Wei, H. L.; Yang, Z.; Chen, Y.; Chu, H. J.; Zhu, J.; Li, Z. C. Eur. Polym. J. 2010, 46, 1032.

[99] Huang, M. N.; Wang, Y. L.; Luo, Y. F. J. Appl. Polym. Sci. 2010, 118,3460 .

[100] Lv, S. H.; Gao, R.; Duan, J.; Cao, Q.; Li, D. J. Appl. Polym. Sci. 2012, 125, 396.

[101] Guo, W.; Sun, N.; Qin, J.; Zhang, J.; Pei, M.; Wang, Y.; Wang, S. J. Appl. Polym. Sci. 2012, 125, 283.

[102] Lv, S.; Ju, H.; Qiu, C.; Ma, Y.; Zhou, Q. J. Appl. Polym. Sci. 2013, $10,3926$.

[103] Habbaba, A.; Lange, A.; Plank, J. J. Appl. Polym. Sci. 2013, 10, 346.

[104] Yang, G. C.; Chen, Z. X.; Zhang, Z. J. React. Funct. Polym. 2002, 51,1 .

[105] Chandrasekhar, S.; Sultana, S.; Narsihmulu, C.; Yadav, J. S.; Gree, R.; Guillemin, J. C. Tetrahedron Lett. 2002, 43, 8335.

[106] Chen, C. P.; Chen, Y. J. Tetrahedron Lett. 2004, 45, 113.

[107] Wang, B. T.; Zhang, Y.; Guo, Z. H.; Cheng, J.; Fang, Z. P. J. Polym. Res. 2011, 18, 187.

[108] Zhao, L. L.; Zhu, L. J.; Wang, Q.; Li, J. L.; Zhang, C. L.; Liu, J. G.; Qu, X. Z.; He, G. L.; Lu, Y. F.; Yang, Z. Z. Soft Matter 2011, 7, 6144.

[109] Erocle, F.; Thissen, H.; Tsang, K.; Evans, R. A.; Forsythe, J. S. Macromolecules 2012, 45, 8387. 